\title{
Optimally adapted meshes for finite elements of arbitrary order and $W^{1, p}$ norms
}

\author{
Jean-Marie Mirebeau
}

October 30, 2018

\begin{abstract}
Given a function $f$ defined on a bounded polygonal domain $\Omega \subset \mathbb{R}^{2}$ and a number $N>0$, we study the properties of the triangulation $\mathcal{T}_{N}$ that minimizes the distance between $f$ and its interpolation on the associated finite element space, over all triangulations of at most $N$ elements. The error is studied in the $W^{1, p}$ semi-norm for $1 \leq p<\infty$, and we consider Lagrange finite elements of arbitrary polynomial order $m-1$. We establish sharp asymptotic error estimates as $N \rightarrow+\infty$ when the optimal anisotropic triangulation is used. A similar problem has been studied in 4, 11, 8, 12, 18, but with the error measured in the $L^{p}$ norm. The extension of this analysis to the $W^{1, p}$ norm is required in order to match more closely the needs of numerical PDE analysis, and it is not straightforward. In particular, the meshes which satisfy the optimal error estimate are characterized by a metric describing the local aspect ratio of each triangle and by a geometric constraint on their maximal angle, a second feature that does not appear for the $L^{p}$ error norm. Our analysis also provides with practical strategies for designing meshes such that the interpolation error satisfies the optimal estimate up to a fixed multiplicative constant.
\end{abstract}

Key words : anisotropic finite elements, $W^{1, p}$ norm, adaptive meshes, interpolation, nonlinear approximation.

AMS subject classifications : $65 \mathrm{D} 05,65 \mathrm{~N} 15,65 \mathrm{~N} 50$

\section{Introduction}

In finite element approximation, a usual distinction is between uniform and adaptive methods. In the latter, the elements defining the mesh may vary strongly in size and shape for a better adaptation to the local features of the approximated function $f$. Such procedures are used to improve the efficiency of numerous numerical methods in scientific computing. This naturally raises the objective of characterizing and constructing an optimal mesh for a given function $f$.

In this paper we consider a bounded bidimensional polygonal domain $\Omega \subset \mathbb{R}^{2}$, a fixed integer $m \geq 2$ and an exponent $1 \leq p<\infty$. For a given conforming triangulation $\mathcal{T}$ of $\Omega$ we denote by $\mathrm{I}_{\mathcal{T}}^{m-1}$ the standard interpolation operator on the space of Lagrange finite elements of degree $m-1$ associated to $\mathcal{T}$. A general objective is to study, for any $f \in C^{m}(\bar{\Omega})$, the optimization problem

$$
\inf _{\#(\mathcal{T}) \leq N}\left\|\nabla\left(f-\mathrm{I}_{\mathcal{T}}^{m-1} f\right)\right\|_{L^{p}(\Omega)}
$$

where the minimum is taken over all (possibly anisotropic) triangulations of cardinality $\leq N$. All the triangulations considered in this paper are assumed to be conforming: they have no hanging nodes, which implies that the interpolant $\mathrm{I}_{\mathcal{T}}^{m-1} f$ is continuous and thus belongs to $W^{1, \infty}(\Omega)$. The choice of the $W^{1, p}$ semi-norm appearing in the expression (1) is motivated by PDE analysis, e.g. elliptic equations in the case $p=2$. Yet our paper in mainly a contribution to approximation theory, and aims at characterizing the approximation power of finite elements on anisotropic meshes. We obtain sharp estimates of the asymptotical behavior of the quantity (1) as $N \rightarrow \infty$, in terms of the $m$-th derivatives of $f$. We also describe practical strategies for constructing meshes that behave similar to the optimal one, in the sense that they asymptotically satisfy as $N \rightarrow \infty$ this sharp error estimate up to a fixed multiplicative constant. Obtaining similar estimates and constructions in a non asymptotic setting remains an open question. 
Estimates of a similar asymptotical nature were obtained in [11, 4, 17] in the particular case of linear finite elements and with the error measured in the $L^{p}$ norm, instead of the $W^{1, p}$ semi-norm. They have the form

$$
\limsup _{N \rightarrow+\infty}\left(N \min _{\#(\mathcal{T}) \leq N}\left\|f-\mathrm{I}_{\mathcal{T}}^{1} f\right\|_{L^{p}(\Omega)}\right) \leq C\left\|\sqrt{\left|\operatorname{det}\left(d^{2} f\right)\right|}\right\|_{L^{p /(p+1)}(\Omega)},
$$

which reveals that the convergence rate is governed by the quantity $\sqrt{\left|\operatorname{det}\left(d^{2} f\right)\right|}$, which depends nonlinearly on the Hessian $d^{2} f$. This is heavily tied to the fact that we allow triangles with possibly highly anisotropic shape. The convergence estimate 22 has been extended to arbitrary approximation order in [18, where the quantity governing the convergence rate for finite elements of arbitrary degree $m-1$ was identified. This quantity depends nonlinearly on the $m$-th order derivative $d^{m} f$. See also the book chapter 12 for an introduction to the subject of adaptive and anisotropic piecewise polynomial approximation.

\section{Main results and layout}

The Taylor development of a function $f \in C^{m}(\bar{\Omega})$, close to a point $z \in \Omega$, can be written under the form

$$
f(z+h)=\mu_{z}(h)+\pi_{z}(h)+o\left(|h|^{m}\right),
$$

where $h \in \mathbb{R}^{2}$ is small, and where $\mu_{z}$ and $\pi_{z}$ are polynomials which respectively belong to the spaces

$$
\mathbb{P}_{m-1}:=\operatorname{Span}\left\{x^{k} y^{l} ; k+l \leq m-1\right\} \quad \text { and } \quad \mathbb{H}_{m}:=\operatorname{Span}\left\{x^{k} y^{l} ; k+l=m\right\} .
$$

For any triangle $T$, we denote by $\mathrm{I}_{T}^{m-1}$ the local interpolation operator acting from $C^{0}(T)$ onto $\mathbb{P}_{m-1}$. For any continuous fonction $\nu \in C^{0}(T)$, the interpolating polynomial $\mathrm{I}_{T}^{m-1} \nu \in \mathbb{P}_{m-1}$ is defined by the conditions

$$
\mathrm{I}_{T}^{m-1} \nu(\gamma)=\nu(\gamma)
$$

for all points $\gamma \in T$ with barycentric coordinates in the set $\left\{0, \frac{1}{m-1}, \cdots, 1\right\}$. If $T$ is a sufficiently small triangle containing the point $z$, we thus have at least heuristically on $T$

$$
\nabla\left(f-\mathrm{I}_{T}^{m-1} f\right) \simeq \nabla\left(\pi_{z}-\mathrm{I}_{T}^{m-1} \pi_{z}\right),
$$

since the Lagrange interpolation operator $\mathrm{I}_{T}^{m-1}$ on the triangle $T$ reproduces the elements of $\mathbb{P}_{m-1}$.

A key ingredient in this paper is the shape function $L_{m, p}$, which is defined by a shape optimization problem: for any $\pi \in \mathbb{H}_{m}$, we define

$$
L_{m, p}(\pi):=\inf _{|T|=1}\left\|\nabla\left(\pi-\mathrm{I}_{T}^{m-1} \pi\right)\right\|_{L^{p}(T)},
$$

where the infimum is taken over all triangles of area $|T|=1$. The solution to this optimization problem thus describes the shape of the triangles of area 1 which are best adapted to the polynomial $\pi$ in the sense of minimizing the interpolation error measured in $W^{1, p}$. If $\mathcal{T}$ is a triangulation of a domain $\Omega$, then $\mathrm{I}_{\mathcal{T}}^{m-1}$ refers to the interpolation operator which coincides with $\mathrm{I}_{T}^{m-1}$ on each $T \in \mathcal{T}$. In view of (5), the optimization problem appearing in (6) can be regarded as a "local" version of the "global" problem (1) of interest.

The function $L_{m, p}$ is the natural generalisation of the function $K_{m, p}$ introduced in 18 for the study of optimal anisotropic triangulations in the sense of the $L^{p}$ interpolation error: for all $\pi \in \mathbb{H}_{m}$

$$
K_{m, p}(\pi):=\inf _{|T|=1}\left\|\pi-\mathrm{I}_{T}^{m-1} \pi\right\|_{L^{p}(\Omega)} .
$$

Throughout this paper we denote by $\tau \in(0, \infty)$ the exponent defined by

$$
\frac{1}{\tau}:=\frac{m-1}{2}+\frac{1}{p} .
$$

Consider two triangles $T, T^{\prime}$ and a polynomial $\pi \in \mathbb{H}_{m}$. If $T$ is mapped onto $T^{\prime}$ by a transformation of the form $z \mapsto \alpha z+\beta$, where $\alpha \in \mathbb{R} \backslash\{0\}$ and $\beta \in \mathbb{R}^{2}$ (in other words the composition of a translation, an 
homothety, and a central symmetry if $\alpha<0$ ), then recalling that $\pi$ is $m$-homogeneous one easily checks that

$$
|T|^{-\frac{1}{\tau}}\left\|\nabla\left(\pi-\mathrm{I}_{T}^{m-1} \pi\right)\right\|_{L^{p}(T)}=\left|T^{\prime}\right|^{-\frac{1}{\tau}}\left\|\nabla\left(\pi-\mathrm{I}_{T^{\prime}}^{m-1} \pi\right)\right\|_{L^{p}\left(T^{\prime}\right)} .
$$

Therefore $\left\|\nabla\left(\pi-\mathrm{I}_{T}^{m-1} \pi\right)\right\|_{L^{p}(T)} \geq|T|^{\frac{1}{\tau}} L_{m, p}(\pi)$ for any triangle $T$ and any $\pi \in \mathbb{H}_{m}$. Our asymptotic error estimate for the optimal triangulation is given by the following theorem.

Theorem 1. For any bounded polygonal domain $\Omega \subset \mathbb{R}^{2}$, any function $f \in C^{m}(\bar{\Omega})$, and any $1 \leq p<\infty$, there exists a sequence of triangulations $\left(\mathcal{T}_{N}\right)_{N \geq N_{0}}$ of $\Omega$, with $\#\left(\mathcal{T}_{N}\right) \leq N$, such that

$$
\limsup _{N \rightarrow \infty} N^{\frac{m-1}{2}}\left\|\nabla\left(f-\mathrm{I}_{\mathcal{T}_{N}}^{m-1} f\right)\right\|_{L^{p}(\Omega)} \leq\left\|L_{m, p}\left(\frac{d^{m} f}{m !}\right)\right\|_{L^{\tau}(\Omega)} .
$$

This theorem is the consequence of a sharper result, Theorem 2 , which is given below. In the above estimate we slightly abuse notations by defining for each $z \in \Omega$

$$
L_{m, p}\left(\frac{d^{m} f(z)}{m !}\right):=L_{m, p}\left(\pi_{z}\right),
$$

where the polynomial $\pi_{z} \in \mathbb{H}_{m}$ is defined by (3). In other words we identify the collection $d^{m} f(z)$ of $m$-th derivatives of $f$ at a given point $z \in \Omega$ to the corresponding term in the Taylor development of $f$ close to $z$. Explicitly the right hand side of (9) stands for

$$
\left\|L_{m, p}\left(\frac{d^{m} f}{m !}\right)\right\|_{L^{\tau}(\Omega)}:=\left(\int_{\Omega} L_{m, p}\left(\pi_{z}\right)^{\tau} d z\right)^{\frac{1}{\tau}} .
$$

The integer $N_{0}$ appearing in Theorem 1 is independent of $f$ and refers to the minimal cardinality of a conforming triangulation of $\Omega$. An important feature of the estimate 9 ) is the "limsup". Recall that the upper limit of a sequence $\left(u_{N}\right)_{N \geq N_{0}}$ is defined by

$$
\limsup _{N \rightarrow \infty} u_{N}:=\lim _{N \rightarrow \infty} \sup _{n \geq N} u_{n}
$$

and is in general stricly smaller than the supremum $\sup _{N>N_{0}} u_{N}$. It is still an open question to find an appropriate upper estimate of $\sup _{N \geq N_{0}} N^{\frac{m-1}{2}}\left\|\nabla\left(f-\mathrm{I}_{\mathcal{T}_{N}}^{m-1} f\right)\right\|_{L^{p}(\Omega)}$ when optimally adapted anisotropic triangulations are used.

We show in $\$ 1$ that a triangulation satisfies the optimal estimate of Theorem 1 up to a fixed multiplicative constant, if it obeys the following four general principles:

(i) The interpolation error should be evenly distributed on all triangles.

(ii) The triangles should adopt locally a specific aspect ratio, dictated by the local value of $d^{m} f$.

(iii) The largest angle of the triangles should be bounded away from $\boldsymbol{\pi}=3.14159 \ldots$

(iv) The triangulation $\mathcal{T}$ should be sufficiently refined in order to adapt to the local features of $f$.

The third point (iii) is the main new ingredient of this paper compared to [18, and is necessary for obtaining optimal $W^{1, p}$ error estimates (but not for $L^{p}$ error estimates). Roughly speaking, two triangles having the same optimized aspect ratio imposed by (ii) may greatly differ in term of their largest angle, and the most acute triangle should be preferred when error is measured in $W^{1, p}$ rather than $L^{p}$. The influence of large angles in mesh adaptation has already been studied in [6, 15, 21, 9]. The heuristic guideline is that large angles should be avoided in general, since they lead to oscillations of the gradient of the interpolant. On the contrary, extremely thin triangles and very small angles can be necessary for optimal mesh adaptation.

The shape function $L_{m, p}$ plays an important role in our results, and we therefore devote $\$ 2$ to its study which is based on algebraic techniques. We obtain explicit minimizers, up to a fixed multiplicative constant, of the optimization problems which correspond to piecewise linear and piecewise quadratic finite element approximation. We also introduce, for arbitrary $m \geq 2$, explicit functions $\pi \in \mathbb{H}_{m} \mapsto \mathbf{L}_{m}(\pi)$ which are defined as the root of a polynomial in the coefficients of $\pi$, and are uniformly equivalent to the shape function $L_{m, p}$, leading therefore to asymptotic error estimates similar to (9) up to multiplicative 
constants.

In order to illustrate the sharpness of Theorem 1, we introduce a slight restriction on sequences of triangulations, following an idea in [4]: a sequence $\left(\mathcal{T}_{N}\right)_{N \geq N_{0}}$ of triangulations is said to be admissible if $\#\left(\mathcal{T}_{N}\right) \leq N$ and $\sup _{N \geq N_{0}}\left(N^{\frac{1}{2}} \sup _{T \in \mathcal{T}_{N}} \operatorname{diam}(T)\right)<\infty$, in other words if

$$
\sup _{T \in \mathcal{T}_{N}} \operatorname{diam}(T) \leq C_{A} N^{-\frac{1}{2}}
$$

for some constant $C_{A}>0$ independent of $N$. Here and below we denote the diameter of a set $E \subset \mathbb{R}^{2}$ by $\operatorname{diam}(E):=\sup \{|x-y| ; x, y \in E\}$. The following theorem shows that the estimate (9) cannot be improved when we restrict our attention to admissible sequences of triangulations. It also shows that this class is reasonably large in the sense that $\sqrt{9}$ is ensured to hold up to small perturbation.

Theorem 2. Let $\Omega \subset \mathbb{R}^{2}$ be a bounded polygonal domain, let $f \in C^{m}(\bar{\Omega})$ and let $1 \leq p<\infty$. For any admissible sequence $\left(\mathcal{T}_{N}\right)_{N \geq N_{0}}$ of triangulations of $\Omega$, one has

$$
\liminf _{N \rightarrow \infty} N^{\frac{m-1}{2}}\left\|\nabla\left(f-\mathrm{I}_{\mathcal{T}_{N}^{m-1}}^{m} f\right)\right\|_{L^{p}(\Omega)} \geq\left\|L_{m, p}\left(\frac{d^{m} f}{m !}\right)\right\|_{L^{\tau}(\Omega)} .
$$

Furthermore, for all $\varepsilon>0$ there exists an admissible sequence of triangulations $\left(\mathcal{T}_{N}^{\varepsilon}\right)_{N \geq N_{0}}$ such that

$$
\limsup _{N \rightarrow \infty} N^{\frac{m-1}{2}}\left\|\nabla\left(f-\mathrm{I}_{\mathcal{T}_{N}^{\varepsilon}}^{m-1} f\right)\right\|_{L^{p}(\Omega)} \leq\left\|L_{m, p}\left(\frac{d^{m} f}{m !}\right)\right\|_{L^{\tau}(\Omega)}+\varepsilon
$$

Note that the sequences of triangulations $\left(\mathcal{T}_{N}^{\varepsilon}\right)_{N \geq N_{0}}$ satisfy the admissibility condition (11) with a constant $C_{A}(\varepsilon)$ which may grow to $+\infty$ as $\varepsilon \rightarrow 0$. Theorem 1 can be inferred from the estimate (13) proceeding as follows: for each $N \geq N_{0}$ and each $\varepsilon>0$ we define a real $\delta(N, \varepsilon)$ by the equality

$$
N^{\frac{m-1}{2}}\left\|\nabla\left(f-\mathrm{I}_{\mathcal{T}_{N}^{\varepsilon}}^{m-1} f\right)\right\|_{L^{p}(\Omega)}=\left\|L_{m, p}\left(\frac{d^{m} f}{m !}\right)\right\|_{L^{\tau}(\Omega)}+\delta(N, \varepsilon) .
$$

We next observe that for any fixed $\varepsilon>0$ one has $\lim \sup _{N \rightarrow \infty} \delta(N, \varepsilon) \leq \varepsilon$. We may therefore construct, using a diagonal extraction procedure, a sequence $\left(\varepsilon_{N}\right)_{N \geq N_{0}}$ such that $\lim _{\sup _{N \rightarrow \infty}} \delta\left(N, \varepsilon_{N}\right) \leq 0$. The sequence of triangulations $\left(\mathcal{T}_{N}^{\varepsilon_{N}}\right)_{N \geq N_{0}}$ then clearly satisfies (9), which establishes Theorem 1

The proof of Theorem 2 is given in $\$ 3$. The proof of the upper estimate 13 involves the construction of an optimal mesh based on a patching strategy adapted from the one encountered in [4. However, inspection of the proof reveals that this construction only becomes effective as the number of triangles $N$ becomes very large. The construction described in $\$$ should therefore be preferred in practical applications.

\section{Notations}

We denote by $\langle u, v\rangle$ the inner product of two vectors $u, v \in \mathbb{R}^{2}$, and by $|u|:=\sqrt{\langle u, u\rangle}$ the euclidean norm of $u$. When $g \in L^{p}\left(\Omega, \mathbb{R}^{2}\right)$ is a vector valued function, we denote by $\|g\|_{L^{p}(\Omega)}$ the $L^{p}$ norm of $x \mapsto|g(x)|$ on $\Omega$, for instance in $(1)$ and $(6)$.

We denote by $\mathrm{M}_{2}$ the set of all $2 \times 2$ real matrices, equipped with the spectral norm $\|A\|:=$ $\max _{|u| \leq 1}|A u|$. We denote by $\mathrm{GL}_{2} \subset \mathrm{M}_{2}$ the group of invertible matrices, by $\mathrm{SL}_{2} \subset \mathrm{GL}_{2}$ the special group of matrices of determinant 1 , and by $\mathcal{O}_{2} \subset \mathrm{GL}_{2}$ the group of orthogonal matrices. We denote by $S_{2} \subset \mathrm{M}_{2}$ the linear space of symmetric matrices, by $S_{2}^{\oplus} \subset S_{2}$ the subset of non-negative symmetric matrices, and by $S_{2}^{+} \subset S_{2}^{\oplus}$ the subset of positive definite symmetric matrices.

For any two symmetric matrices $S, S^{\prime} \in S_{2}$, we write $S \leq S^{\prime}$ if and only if $S^{\prime}-S \in S_{2}^{\oplus}$. For any $S \in S_{2}^{\oplus}\left(\right.$ resp. $\left.S_{2}^{+}\right)$and any $\alpha>0$ (resp. $\alpha \in \mathbb{R}$ ) we denote by $S^{\alpha}$ the symmetric matrix obtained by elevating the eigenvalues to the power $\alpha$ in a diagonalization of $S$.

The greek letter $\pi$ always refers to an homogeneous polynomial $\pi \in \mathbb{H}_{m}$, while the bold notation $\pi$ refers to the mathematical constant $\boldsymbol{\pi}=3.14159 \ldots$ 


\section{Adaptive mesh generation}

This section describes some properties that are needed to ensure that a mesh $\mathcal{T}$ satisfies the optimal error estimate introduced in Theorem 1 up to a fixed multiplicative constant. We first introduce a description of the triangle based on some parameters adapted to our purposes. We then prescribe the behavior of these parameters in a triangulation tailored to the approximation of a given function $f$, and we discuss the practical challenges encountered in the construction of such a mesh.

\subsection{Description of a triangle}

A triangle $T \subset \mathbb{R}^{2}$ is determined by the collection of its three vertices $v_{1}, v_{2}$ and $v_{3}$. In the context of adaptive mesh generation, we rather adopt the following parameters. The position of $T$ is determined by its barycenter

$$
z_{T}:=\left(v_{1}+v_{2}+v_{3}\right) / 3
$$

The area, the aspect ratio and the orientation of $T$ are encoded in a symmetric positive definite matrix $\mathcal{H}_{T} \in S_{2}^{+}$defined by the equality

$$
\mathcal{H}_{T}^{-1}:=\frac{2}{3} \sum_{1 \leq i \leq 3}\left(v_{i}-z_{T}\right)\left(v_{i}-z_{T}\right)^{\mathrm{T}} .
$$

Last we shall introduce below a real $S(T) \geq 1$ which is tied to the largest angle of $T$.

If a triangle $T^{\prime}$ is mapped onto $T$ by the change of coordinates $z \mapsto A z+z_{0}$, where $A \in \mathrm{GL}_{2}$ and $z_{0} \in \mathbb{R}^{2}$, then one easily checks that

$$
\mathcal{H}_{T^{\prime}}=A^{\mathrm{T}} \mathcal{H}_{T} A
$$

From this point onwards we denote by $T_{\text {eq }}$ the triangle of vertices $(\cos (2 k \pi / 3), \sin (2 k \pi / 3))_{0 \leq k \leq 2}$, which satisfies $\mathcal{H}_{T_{\mathrm{eq}}}=\mathrm{Id}$. Combining this observation with [14, Proposition 5.1.3 in [20] establishes that for any triangle $T$

$$
|T| \sqrt{\operatorname{det} \mathcal{H}_{T}}=\left|T_{\text {eq }}\right|
$$

and that there exists a rotation $U \in \mathcal{O}_{2}$ (depending on $T$ ) such that the change of coordinates

$$
z \mapsto U \mathcal{H}_{T}^{\frac{1}{2}}\left(z-z_{T}\right)
$$

maps $T$ onto $T_{\text {eq }}$. Furthermore, as illustrated on Figure 1 (left), we have the inclusions

$$
\left\{z_{T}+u ; u^{\mathrm{T}} \mathcal{H}_{T} u \leq 1 / 4\right\} \subset T \subset \mathcal{E}_{T}:=\left\{z_{T}+u ; u^{\mathrm{T}} \mathcal{H}_{T} u \leq 1\right\} .
$$

The inclusion of two triangles $T, T^{\prime}$ therefore implies an inclusion of ellipses, hence an inequality on the associated symmetric matrices:

$$
T^{\prime} \subset T \Rightarrow 4 \mathcal{H}_{T^{\prime}} \geq \mathcal{H}_{T}
$$

We denote by $\rho(T) \in[1, \infty[$ the measure of degeneracy of a triangle $T$, which is defined as follows:

$$
\rho(T):=\sqrt{\left\|\mathcal{H}_{T}\right\|\left\|\mathcal{H}_{T}^{-1}\right\|}
$$

Lemma 1.1. For each triangle $T$, one has

$$
\rho(T) \leq \frac{\operatorname{diam}(T)^{2}}{|T| /\left|T_{\mathrm{eq}}\right|} \leq 4 \rho(T)
$$

Proof. It follows from 15 that $|T| /\left|T_{\text {eq }}\right|=\left\|\mathcal{H}_{T}\right\|^{-\frac{1}{2}}\left\|\mathcal{H}_{T}^{-\frac{1}{2}}\right\|$, and from 17 that $\left\|\mathcal{H}_{T}^{-\frac{1}{2}}\right\|=\operatorname{diam}\left(\mathcal{E}_{T}\right) / 2 \leq$ $\operatorname{diam}(T) \leq \operatorname{diam}\left(\mathcal{E}_{T}\right)$. Combining this with $(19)$ we obtain the announced result. 

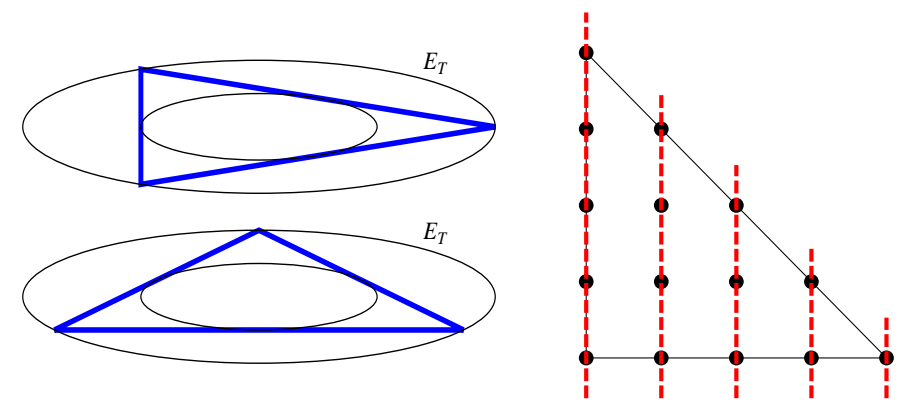

Figure 1: Two triangles and the associated ellipses associated by (17) (left). The interpolation points on the triangle $T_{0}$ are aligned vertically (right).

As illustrated on Figure 1, the fact that a triangle $T$ is acute, or not, is not reflected on the ellipsoid $\mathcal{E}_{T}$ or the matrix $\mathcal{H}_{T}$. Since acute triangles play a priviledged role in finite element approximation we introduce the measure of sliverness $S(T)$ of a triangle $T$, which is defined as follows

$$
S(T):=\inf \left\{\|\psi\|\left\|\psi^{-1}\right\| ; \psi \in \mathrm{GL}_{2} \text { s.t. the image of } T \text { by } \psi \text { is acute }\right\} .
$$

(Where we refer to the image of $T$ by the linear change of coordinates $z \mapsto \psi z$ on $\mathbb{R}^{2}$.) The quantity $S(T)$ can be regarded as the distance from $T$ to the collection of acute simplices. It immediately follows from 16 and 19 that

$$
1 \leq S(T) \leq \rho(T) .
$$

Then next expression gives an explicit expression of $S(T)$ in terms of the largest angle of $T$, which shows that it is equivalent up to a multiplicative constant to the quantities previously introduced in [2, 15, and referred to as $\sigma_{\min }(T)$ and $1 / \cos \left(\theta^{\prime}\right)$ respectively.

Proposition 1.2. For any triangle $T$ with largest interior angle $\theta$, one has $S(T)=\max \left\{1, \tan \frac{\theta}{2}\right\}$.

Proof. The result of this proposition is trivial if the triangle $T$ is acute, we therefore assume that $T$ is obtuse. We assume without loss of generality that the vertices of $T$ are $0, \alpha u$ and $\beta v$, where $\alpha, \beta>0$, $u, v \in \mathbb{R}^{2},|u|=|v|=1$ and $\langle u, v\rangle=\cos \theta$. Note that $|u-v|=2 \sin (\theta / 2)$ and $|u+v|=2 \cos (\theta / 2)$. Let $\psi \in \mathrm{GL}_{2}$ be such that the image of $T$ by $\psi$ is acute. We thus have $\langle\psi(u), \psi(v)\rangle \geq 0$ and therefore $|\psi(u)-\psi(v)| \leq|\psi(u)+\psi(v)|$. It follows that

$$
\|\psi\|\left\|\psi^{-1}\right\| \geq \frac{|u-v|}{|u+v|} \times \frac{|\psi(u)+\psi(v)|}{|\psi(u)-\psi(v)|} \geq \frac{2 \sin (\theta / 2)}{2 \cos (\theta / 2)}=\tan \frac{\theta}{2} .
$$

Therefore $S(T) \geq \tan \frac{\theta}{2}$. On the other hand, let $\psi$ be defined by $\psi(u)=(0,1)$ and $\psi(v)=(1,0)$. Obviously the image of $T$ by $\psi$ has one of its angles equal to $\boldsymbol{\pi} / 2$, and is therefore acute. One easily checks that $\|\psi\|\left\|\psi^{-1}\right\|=\tan (\theta / 2)$ and therefore $S(T) \leq \tan \frac{\theta}{2}$, which concludes the proof of this proposition.

Most error estimates available in the literature, such as in [6, 15, are designed to control the gradient interpolation error of a function on a triangle, by second or higher derivatives of the approximated function. Our purposes require a slight variant of these estimates, given in the next lemma, in which the gradient interpolation error is controlled by the gradient itself of the approximated function.

Lemma 1.3. There exists a constant $C=C(m)$ such that the following holds. For any triangle $T$ and any $f \in W^{1, \infty}(T)$, one has

$$
\left\|\nabla\left(f-\mathrm{I}_{T}^{m-1} f\right)\right\|_{L^{\infty}(T)} \leq C S(T)\|\nabla f\|_{L^{\infty}(T)} .
$$

Proof. Let $T_{0}$ be the triangle of vertices $(0,0),(1,0)$ and $(0,1)$, and let $g \in W^{1, \infty}\left(T_{0}\right)$. We define $\tilde{g}(x, y):=$ $g(x, 0)$ and $h(x, y):=g(x, y)-g(x, 0)$. Since $\tilde{g}$ does not depend on $y$ and since the Lagrange interpolation points on $T_{0}$ are aligned vertically, as illustrated on Figure 1 , the Lagrange interpolant $\mathrm{I}_{T_{0}}^{m-1} \tilde{g}$ does not 
depend on $y$ either. Futhermore, for all $(x, y) \in T_{0}$, we have $|h(x, y)|=\left|\int_{s=0}^{y} \frac{\partial g}{\partial y}(x, s) d s\right| \leq\left\|\frac{\partial g}{\partial y}\right\|_{L^{\infty}\left(T_{0}\right)}$. Hence

$$
\left\|\frac{\partial \mathrm{I}_{T_{0}}^{m-1} g}{\partial y}\right\|_{L^{\infty}\left(T_{0}\right)}=\left\|\frac{\partial \mathrm{I}_{T_{0}}^{m-1} h}{\partial y}\right\|_{L^{\infty}\left(T_{0}\right)} \leq C_{0}\left\|\mathrm{I}_{T_{0}}^{m-1} h\right\|_{L^{\infty}\left(T_{0}\right)} \leq C_{0} C_{1}\|h\|_{L^{\infty}\left(T_{0}\right)} \leq C_{0} C_{1}\left\|\frac{\partial g}{\partial y}\right\|_{L^{\infty}\left(T_{0}\right)},
$$

where the constants $C_{0}$ and $C_{1}$ are the $L^{\infty}\left(T_{0}\right)$ norms of the operators $g \in \mathbb{P}_{m-1} \mapsto \frac{\partial g}{\partial y} \in \mathbb{P}_{m-2}$, and $g \in C^{0}\left(T_{0}\right) \mapsto \mathrm{I}_{T_{0}}^{m-1} g \in \mathbb{P}_{m-1}$ respectively.

Let $e$ be an edge vector of the triangle $T$. There exists an affine change of coordinates $\Psi$ on $\mathbb{R}^{2}$, with linear part $\psi \in \mathrm{GL}_{2}$, such that $\Psi(T)=T_{0}$ and $\psi e=e_{0}$, where $e_{0}=(0,1)$ is the vertical edge vector of $T_{0}$. Noticing that

$$
\left\langle e, \nabla \mathrm{I}_{T}^{m-1}(g \circ \Psi)\right\rangle=\left\langle e, \nabla\left(\left(\mathrm{I}_{T_{0}}^{m-1} g\right) \circ \Psi\right)\right\rangle=\left\langle e_{0},\left(\nabla \mathrm{I}_{T_{0}}^{m-1} g\right) \circ \Psi\right\rangle=\frac{\partial \mathrm{I}_{T_{0}}^{m-1} g}{\partial y} \circ \Psi,
$$

we obtain

$$
\left\|\left\langle e, \nabla \mathrm{I}_{T}^{m-1}(g \circ \Psi)\right\rangle\right\|_{L^{\infty}(T)}=\left\|\frac{\partial \mathrm{I}_{T_{0}}^{m-1} g}{\partial y}\right\|_{L^{\infty}\left(T_{0}\right)} \leq C_{0} C_{1}\left\|\frac{\partial g}{\partial y}\right\|_{L^{\infty}\left(T_{0}\right)}=C_{0} C_{1}\|\langle e, \nabla(g \circ \Psi)\rangle\|_{L^{\infty}(T)} .
$$

Applying this inequality to $g=f \circ \Psi^{-1}$ we obtain that

$$
\left\|\left\langle e, \nabla \mathrm{I}_{T}^{m-1} f\right\rangle\right\|_{L^{\infty}(T)} \leq C_{0} C_{1}\|\langle e, \nabla f\rangle\|_{L^{\infty}(T)},
$$

for any edge vector $e \in\{a, b, c\}$ of $T$. Defining the norm $|v|_{T}:=\max \{|\langle v, a\rangle| /|a|,|\langle v, b\rangle| /|b|,|\langle v, c\rangle| /|c|\}$, we obtain

$$
\left\|\left|\nabla \mathrm{I}_{T}^{m-1} f\right|_{T}\right\|_{L^{\infty}(T)} \leq C_{0} C_{1}\left\||\nabla f|_{T}\right\|_{L^{\infty}(T)} .
$$

For any $v \in \mathbb{R}^{2}$ one has $\cos (\theta / 2)|v| \leq|v|_{T} \leq|v|$, where $\theta$ denotes the maximal angle of $T$. Indeed the upper inequality is trivial and the lower one is follows from the fact that at least one of the edge vectors makes an angle less than $\theta / 2$ with $v$. Combining this with (24), we obtain

$$
\left\|\nabla \mathrm{I}_{T}^{m-1} f\right\|_{L^{\infty}(T)} \leq \frac{C_{0} C_{1}}{\cos (\theta / 2)}\|\nabla f\|_{L^{\infty}(T)}
$$

Since $\theta \geq \pi / 3$ we have $\frac{1}{\cos (\theta / 2)} \leq 2 \tan (\theta / 2) \leq 2 S(T)$ according to Proposition 1.2 which concludes the proof with $C=2 C_{0} C_{1}+1$.

Remark. The definition (20) of the measure of sliverness $S(T)$, Propositions 1.2 and Lemma 1.3 have analogs for simplices of arbitrary dimension, see for instance the definition (3.87), Proposition 3.6.1 and Lemma 3.6 .3 in [20].

\subsection{Construction of a triangulation adapted to a function}

In order to identify the optimal shape of the triangles, our first step is to reformulate the optimization problem appearing in the definition (6) of the shape function $L_{m, p}$. One easily checks using the invariance property (8) that for any $\pi \in \mathbb{H}_{m}$

$$
L_{m, p}(\pi)=\inf \left\{|T|^{-\frac{m-1}{2}} ; T \text { s.t. }|T|^{-\frac{1}{p}}\left\|\nabla\left(\pi-\mathrm{I}_{T}^{m-1} \pi\right)\right\|_{L^{p}(T)} \leq 1\right\},
$$

and the minimizers of this optimization problem and the original one are homothetic.

The next lemma shows that the exponent $p$ can be disregarded in the above expression, when one is only interested in minimizing it up to a fixed multiplicative constant.

Lemma 1.4. There exists a constant $c=c(m)>0$ such that for any triangle $T$ and any $\pi \in \mathbb{H}_{m}$

$$
c\left\|\nabla\left(\pi-\mathrm{I}_{T}^{m-1} \pi\right)\right\|_{L^{\infty}(T)} \leq|T|^{-\frac{1}{p}}\left\|\nabla\left(\pi-\mathrm{I}_{T}^{m-1} \pi\right)\right\|_{L^{p}(T)} \leq\left\|\nabla\left(\pi-\mathrm{I}_{T}^{m-1} \pi\right)\right\|_{L^{\infty}(T)} .
$$


Proof. We denote by $T_{0}$ an arbitrary but fixed triangle of area 1 . For all $g \in L^{\infty}\left(T_{0}, \mathbb{R}^{2}\right)$ we obtain using Jensen's inequality

$$
\|g\|_{L^{1}\left(T_{0}\right)} \leq\|g\|_{L^{p}\left(T_{0}\right)} \leq\|g\|_{L^{\infty}\left(T_{0}\right)} .
$$

Furthermore, since all norms are equivalent on the finite dimensional space $\mathbb{P}_{m-1}^{2}$, there exists a constant $c=c(m)>0$ such that $c\|\mu\|_{L^{\infty}\left(T_{0}\right)} \leq\|\mu\|_{L^{1}\left(T_{0}\right)}$ for all $\mu \in \mathbb{P}_{m-1}^{2}$. Therefore

$$
\begin{aligned}
c\|\mu\|_{L^{\infty}\left(T_{0}\right)} & \leq\|\mu\|_{L^{1}\left(T_{0}\right)} \leq\|\mu\|_{L^{p}\left(T_{0}\right)} \leq\|\mu\|_{L^{\infty}\left(T_{0}\right)}, \\
c\|\mu\|_{L^{\infty}(T)} & \leq|T|^{-1}\|\mu\|_{L^{1}(T)} \leq|T|^{-\frac{1}{p}}\|\mu\|_{L^{p}(T)} \leq\|\mu\|_{L^{\infty}(T)},
\end{aligned}
$$

where we used in the second line a change of variables from $T_{0}$ to an arbitrary triangle $T$. We conclude the proof of this lemma by injecting $\mu=\nabla\left(\pi-\mathrm{I}_{T}^{m-1} \pi\right)$ in 27$)$, where $\pi \in \mathbb{H}_{m}$ is arbitrary.

For each $\mu \in \mathbb{H}_{m-1}^{2}$ we define

$$
\|\mu\|:=\sup _{z \neq 0} \frac{|\mu(z)|}{|z|^{m-1}}
$$

and for each $A \in \mathrm{M}_{2}$ we define $\mu \circ A \in \mathbb{H}_{m-1}^{2}$ by $\mu \circ A(z):=\mu(A z)$. Note that $\|\mu \circ U\|=\|\mu\|$ for any $U \in \mathcal{O}_{2}$, and that for $M \in S_{2}^{+}$

$$
\left\|\mu \circ M^{-\frac{1}{2}}\right\|=\sup _{z \neq 0} \frac{|\mu(z)|}{|z|_{M}^{m-1}} \quad \text { where } \quad|z|_{M}:=\sqrt{z^{\mathrm{T}} M z}
$$

hence for $M, M^{\prime} \in S_{2}^{+}$

$$
M \leq M^{\prime} \Rightarrow\left\|\pi \circ M^{-\frac{1}{2}}\right\| \geq\left\|\pi \circ M^{\prime-\frac{1}{2}}\right\| .
$$

The next lemma shows that, among the triangles having a prescribed aspect ratio, encoded by a matrix $M$, the smallest approximation error is achieved by the acute triangles (up to a fixed multiplicative constant) for which the measure of sliverness $S$ is minimal and equals one.

Lemma 1.5. There exists a constant $C=C(m) \geq 1$ such that the following holds. For any triangle $T$ and any $\pi \in \mathbb{H}_{m}$, one has denoting $M:=\mathcal{H}_{T}$

$$
C^{-1}\left\|(\nabla \pi) \circ M^{-\frac{1}{2}}\right\| \leq\left\|\nabla\left(\pi-\mathrm{I}_{T}^{m-1} \pi\right)\right\|_{L^{\infty}(T)} \leq C S(T)\left\|(\nabla \pi) \circ M^{-\frac{1}{2}}\right\| .
$$

Proof. We may assume that the triangle $T$ is centered at the origin of $\mathbb{R}^{2}$. We obtain combining Lemma 1.3 and the inclusion $T \subset \mathcal{E}_{T}$, see 17,

$$
\left\|\nabla\left(\pi-\mathrm{I}_{T}^{m-1} \pi\right)\right\|_{L^{\infty}(T)} \leq C S(T)\|\nabla \pi\|_{L^{\infty}(T)} \leq C S(T)\|\nabla \pi\|_{L^{\infty}\left(\mathcal{E}_{T}\right)}=C S(T)\left\|(\nabla \pi) \circ M^{-\frac{1}{2}}\right\|,
$$

which establishes the right part of (31). On the other hand, if $C$ is sufficiently large, one has for all $\mu \in \mathbb{H}_{m-1}^{2}$

$$
C^{-1}\|\mu\| \leq \inf _{\nu \in \mathbb{P}_{m-2}^{2}}\|\mu-\nu\|_{L^{\infty}\left(T_{\mathrm{eq}}\right)}
$$

since both quantities are norms on $\mathbb{H}_{m-1}^{2}$. Since $z_{T}=0$, the change of variables $z \mapsto M^{-\frac{1}{2}} U^{\mathrm{T}} z$ maps $T_{\text {eq }}$ onto $T$, where $U \in \mathcal{O}_{2}$ is defined by 16 . Applying the above inequality to $\mu \circ\left(M^{-\frac{1}{2}} U^{\mathrm{T}}\right)$, where $\mu \in \mathbb{H}_{m-1}^{2}$ is arbitrary, we thus obtain

$$
C^{-1}\left\|\mu \circ M^{-\frac{1}{2}}\right\|=C^{-1}\left\|\mu \circ\left(M^{-\frac{1}{2}} U^{\mathrm{T}}\right)\right\| \leq \inf _{\nu \in \mathbb{P}_{m-2}^{2}}\left\|\mu \circ\left(M^{-\frac{1}{2}} U^{\mathrm{T}}\right)-\nu\right\|_{L^{\infty}\left(T_{\mathrm{eq}}\right)}=\inf _{\nu \in \mathbb{P}_{m-2}^{2}}\|\mu-\nu\|_{L^{\infty}(T)} .
$$

Choosing $\mu=\nabla \pi$ and $\nu=\nabla \mathrm{I}_{T}^{m-1} \pi$ we obtain the left part of 31 , which concludes the proof.

We introduce a variant $L_{m}$ of the shape function, following an idea originally proposed in [8]: for each $\pi \in \mathbb{H}_{m}$

$$
L_{m}(\pi):=\inf \left\{(\operatorname{det} M)^{\frac{m-1}{4}} ; M \in S_{2}^{+} \text {s.t. }\|(\nabla \pi) \circ M\| \leq 1\right\}
$$


Lemma 1.6. There exists a constant $C=C(m) \geq 1$ such that for all $\pi \in \mathbb{H}_{m}$

$$
C^{-1} L_{m}(\pi) \leq L_{m, p}(\pi) \leq C L_{m}(\pi) .
$$

Proof. Injecting (15), Lemma 1.4 and Lemma 1.5 into 25) we obtain

$$
\begin{aligned}
& \inf \left\{\left(\left|T_{\text {eq }}\right| /|T|\right)^{\frac{m-1}{2}} ; T \text { s.t. } \quad|T|^{-\frac{1}{p}}\left\|\nabla\left(\pi-\mathrm{I}_{T}^{m-1} \pi\right)\right\|_{L^{p}(T)} \leq C\right\} \\
\leq & \inf \left\{(\operatorname{det} M)^{\frac{m-1}{4}} ; M \in S_{2}^{+} \text {s.t. }\|(\nabla \pi) \circ M\| \leq 1\right\} \\
\leq & \inf \left\{\left(\left|T_{\text {eq }}\right| /|T|\right)^{\frac{m-1}{2}} ; T \text { s.t. }|T|^{-\frac{1}{p}}\left\|\nabla\left(\pi-\mathrm{I}_{T}^{m-1} \pi\right)\right\|_{L^{p}(T)} \leq c / C\right\},
\end{aligned}
$$

where we used the fact that for any $M \in S_{2}^{+}$there exists an acute triangle $T$ such that $\mathcal{H}_{T}=M$. Hence

$$
\left|T_{\mathrm{eq}}\right|^{\frac{m-1}{2}} L_{m, p}(\pi / C) \leq L_{m}(\pi) \leq\left|T_{\mathrm{eq}}\right|^{\frac{m-1}{2}} L_{m, p}(C \pi / c)
$$

which concludes the proof since the shape function satisfies the homogeneity property $L_{m, p}(\lambda \pi)=$ $|\lambda| L_{m, p}(\pi)$ for any $\lambda \in \mathbb{R}$, as can be seen from the expression (6).

Following a suggestion of the referees, we may compare Lemma 1.5 to other anisotropic error estimates that have been proposed in the literature.

Remark 1.7. The specificities of the estimate 31) are the following:

1. It only applies to an exactly polynomial function $f=\pi \in \mathbb{H}_{m}$, or $f=\pi+\mu$ where $\mu \in \mathbb{P}_{m-1}$ since the interpolation operator reproduces these elements: $\mu=\mathrm{I}_{T}^{m-1} \mu$. This is a strong restriction, yet sufficient for our purposes, when compared for instance to [1] which applies to arbitrary functions in an adequate smoothness space.

2. It is sharp (up to the multiplicative constant $C$ ) for any polynomial $\pi \in \mathbb{H}_{m}$ and any acute triangle $T$. The sharpness is lost in the case of a strongly obtuse triangle since the measure of sliverness $S(T)$ only appears on the right of (31). A more elaborate estimate, which is restricted to the case $m=2$ of linear interpolation but applies to general functions, is proposed in [13] in the attempt (confirmed by some examples) to provide a sharp estimate for both acute and obtuse triangles. A detailed study of the interpolation error of a polynomial function, with respect to the shape of the triangle $T$, can also be found in [9, 10] in the cases $m=2$ or $m=3$ respectively.

3. The orientation and the scales of the triangle $T$ are encoded in the matrix $M=\mathcal{H}_{T} \in S_{2}^{+}$. This is one of the major strengths of this estimate, because it leads to the optimization problem (32) posed on the set $S_{2}^{+}$of symmetric positive definite matrices which can be addressed mathematically. This problem is studied in 9 2 using algebraic techniques which yield an explicit equivalent of the shape functions $L_{m, p}$ for all $m \geq 2$, and an explicit "near minimizer" $\pi \in \mathbb{H}_{m} \mapsto \mathcal{M}_{m}(\pi) \in S_{2}^{+}$, for $m \in\{2,3\}$, of the minimization problem (32) defining $L_{m}(\pi)$. This problem is also studied in Chapter 6 of [20] using analytical techniques, which yield well behaved, although implicit, "near minimizers" $\pi \mapsto \mathcal{M}_{m}(\pi)$ of (32) for all $m \geq 2$.

We now focus our attention on the global approximation of a function $f \in C^{m}(\bar{\Omega})$ in the $W^{1, p}$ semi-norm. For that purpose we assume that a map $z \in \bar{\Omega} \mapsto \mathcal{M}(z) \in S_{2}^{+}$has been obtained which satisfies

$$
\int_{\Omega}(\operatorname{det} \mathcal{M}(z))^{\frac{m-1}{4} \tau} d z \leq C_{L}^{\tau} \int_{\Omega} L_{m}\left(\pi_{z}\right)^{\tau} d z, \quad \text { and } \quad\left\|\pi_{z} \circ \mathcal{M}(z)^{-\frac{1}{2}}\right\| \leq 1 \text { for all } z \in \Omega,
$$

where the polynomial $\pi_{z} \in \mathbb{H}_{m}$ is defined by (3), the exponent $\tau$ by $(7)$, and $C_{L}$ is a constant not too large. In other words we assume that the matrix $\mathcal{M}(z)$ is a minimizer, in an average sense and up to the sub-optimality constant $C_{L}$, of the optimization problem appearing in the definition (32) of $L_{m}\left(\pi_{z}\right)$. Such a map can be obtained by setting $\mathcal{M}(z):=\mathcal{M}_{m}\left(\pi_{z}\right)+\delta$ Id, where $\mathcal{M}_{m}$ is described in Point 3 of Remark 1.7 and where $\delta>0$ is a positive constant introduced to avoid degeneracy problems. 

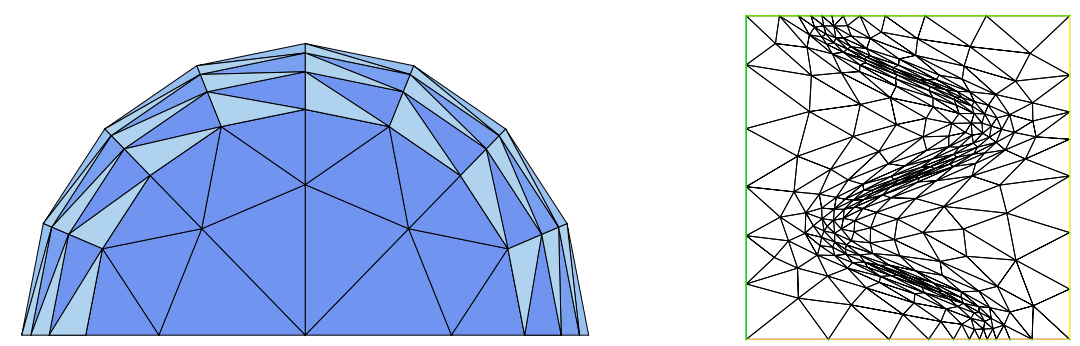

Figure 2: Anisotropic mesh generation with a guaranteed upper bound on the maximal angle of the elements is generally tractable if one only requires some anisotropy close to the boundary of the domain, and tangentially to it (left). This is not any more the case if some anisotropy is required in the interior of the domain, at least with current software such as 22] (right).

We introduce a Riemannian metric $H: \Omega \rightarrow S_{2}^{+}$

$$
H(z):=h^{-2}(\operatorname{det} \mathcal{M}(z))^{\frac{-1}{(m-1) p+2}} \mathcal{M}(z),
$$

where $h>0$ is a parameter. Mesh generation software such as [22], see Figure 2[(right), and [23] in three dimensions (see [17] for a more extensive list), are designed to produce a mesh $\mathcal{T}$ of $\Omega$ such that

$$
C_{0}^{-2} H(z) \leq \mathcal{H}_{T} \leq C_{0}^{2} H(z)
$$

for all $T \in \mathcal{T}, z \in T$, where $C_{0} \geq 1$ is a constant not too large which reflects the quality of the adaptation of the mesh $\mathcal{T}$ to the metric $H$. In the expression (34) of the Riemannian metric $H$, the matrix $\mathcal{M}(z)$ is used to prescribe optimal aspects ratios (requirement ii in the introduction) for the triangles $T \in \mathcal{T}$, and the scalar factor to equidistribute the interpolation errors among the elements of $\mathcal{T}$ (requirement i). Theoretical guarantees for such algorithms were established in [16, 7, 20] when the metric sufficiently regular. Unfortunately these results do not guarantee any property of the measure of sliverness $S(T)$ of the generated triangles $T \in \mathcal{T}$ (requirement iii). This generally forbids to achieve the optimal convergence estimate stated in Theorem 11. even up to a fixed multiplicative constant. The adaptation of the method presented here to this (suboptimal) context is described [19].

Limited results exist nevertheless on anisotropic mesh generation with some control on the measure of sliverness. Such a construction is (usually) possible if one only requires some anisotropy close to the boundary of the domain, and tangentially to it, see Figure 2 (left), which is the adequate behavior for the discretization of number of problems (such as the Poisson equation, or singularly perturbed reaction diffusion problems) as discussed in 22. In the general case where some anisotropy is required in the interior of the domain, we refer to Theorem 6.1.2 in Chapter 6 of [20, on "quasi-acute triangulations", which implies the following. Assume that $\Omega$ is the periodic domain $(\mathbb{R} / \mathbb{Z})^{2}$; the key is the absence of a boundary. Assume that the metric $H$ satisfies the strong regularity properties required for this result, which are expressed under the form of Lipschitz regularity with respect to some distances on $S_{2}^{+}$and $\mathbb{R}^{2}$, and are satisfied if the parameter $h>0$ in (34) is sufficiently small according to Lemma 6.5.12 in [20]. Then there exists a triangulation $\mathcal{T}$ which satisfies (35), for an absolute constant $C_{0}$ independent of $H$, and in addition the following property: there exists a $C_{0}$-refinement $\mathcal{T}^{\prime}$ of the triangulation $\mathcal{T}$ (in other words each element of $\mathcal{T}$ contains at most $C_{0}$ elements of $\mathcal{T}^{\prime}$, and each element of $\mathcal{T}^{\prime}$ is contained in an element of $\mathcal{T}$ ) such that $S\left(T^{\prime}\right) \leq C_{0}$ for all $T^{\prime} \in \mathcal{T}^{\prime}$ (requirement iii in the introduction).

Let $\left(\mathcal{T}, \mathcal{T}^{\prime}\right)$ be a pair of triangulations satisfying the above conditions. If $T(z) \in \mathcal{T}$ denotes the element containing a point $z \in \Omega$, then we obtain using (15) and (35)

$$
\#\left(\mathcal{T}^{\prime}\right) \leq C_{0} \#(\mathcal{T})=C_{0} \int_{\Omega} \frac{d z}{|T(z)|} \leq \frac{C_{0}^{3}}{\left|T_{\mathrm{eq}}\right|} \int_{\Omega} \sqrt{\operatorname{det} H}=\frac{C_{0}^{3}}{\left|T_{\mathrm{eq}}\right|} h^{-2} \int_{\Omega}(\operatorname{det} \mathcal{M})^{\frac{m-1}{4} \tau} .
$$

Let $z \in \Omega, T^{\prime} \in \mathcal{T}^{\prime}$ and $T \in \mathcal{T}$ be such that $z \in T^{\prime} \subset T$. Using 18 and 35 we obtain $\mathcal{H}_{T}^{\prime} \geq 2^{-2} \mathcal{H}_{T} \geq$ 
$\left(2 C_{0}\right)^{-2} H(z)$, hence

$$
\begin{aligned}
\left\|\nabla\left(\pi_{z}-\mathrm{I}_{T^{\prime}}^{m-1} \pi_{z}\right)\right\|_{L^{p}\left(T^{\prime}\right)} & \leq\left|T^{\prime}\right|^{\frac{1}{p}}\left\|\nabla\left(\pi_{z}-\mathrm{I}_{T^{\prime}}^{m-1} \pi_{z}\right)\right\|_{L^{\infty}\left(T^{\prime}\right)} \\
& \leq C\left|T_{\mathrm{eq}}\right|^{\frac{1}{p}}\left(\operatorname{det} \mathcal{H}_{T^{\prime}}\right)^{-\frac{1}{2 p}} S\left(T^{\prime}\right)\left\|\left(\nabla \pi_{z}\right) \circ \mathcal{H}_{T^{\prime}}^{-\frac{1}{2}}\right\| \\
& \leq C_{1}(\operatorname{det} H(z))^{-\frac{1}{2 p}}\left\|\left(\nabla \pi_{z}\right) \circ H(z)^{-\frac{1}{2}}\right\| \\
& =C_{1} h^{\frac{2}{\tau}}\left\|\left(\nabla \pi_{z}\right) \circ \mathcal{M}(z)^{-\frac{1}{2}}\right\| \\
& \leq C_{1} h^{\frac{2}{\tau}}
\end{aligned}
$$

where $C_{1}:=C\left|T_{\mathrm{eq}}\right|^{\frac{1}{p}} 2^{\frac{1}{\tau}} C_{0}^{\frac{1}{\tau}+1}$. We used successively Jensen's inequality in the first line, 15 and Lemma 1.5 in the second line, $S\left(T^{\prime}\right) \leq C_{0}$ the definition (7) of $\tau$ and (30) in the third line, (34) in the fourth line and (33) in the last line. In order to control the function $f$, instead of the polynomial $\pi_{z}$ which corresponds to its Taylor expansion, we may use Point i of Lemma 3.1. proven in $\$ 3$ below, which immediately implies in this context that

$$
\left|\left\|\nabla\left(f-\mathrm{I}_{T^{\prime}}^{m-1} f\right)\right\|_{L^{p}\left(T^{\prime}\right)}-\left\|\nabla\left(\pi_{z}-\mathrm{I}_{T^{\prime}}^{m-1} \pi_{z}\right)\right\|_{L^{p}\left(T^{\prime}\right)}\right| \leq \varepsilon(h) h^{\frac{2}{\tau}}
$$

where $\varepsilon(h) \rightarrow 0$ as $h \rightarrow 0$, and the function $\varepsilon: \mathbb{R}_{+}^{*} \rightarrow \mathbb{R}_{+}$only depends on $f, m$ and $\mathcal{M}$. If $h$ is sufficiently small (requirement iv in the introduction), then $\varepsilon(h) \leq 1$ and therefore $\left\|\nabla\left(f-\mathrm{I}_{T^{\prime}}^{m-1} f\right)\right\|_{L^{p}\left(T^{\prime}\right)} \leq\left(C_{1}+1\right) h^{\frac{2}{\tau}}$ for each $T^{\prime} \in \mathcal{T}^{\prime}$. It follows that

$$
\begin{aligned}
\#\left(\mathcal{T}^{\prime}\right)^{\frac{m-1}{2}}\left\|\nabla\left(f-\mathrm{I}_{\mathcal{T}^{\prime}}^{m-1} f\right)\right\|_{L^{p}(\Omega)} & \leq \#\left(\mathcal{T}^{\prime}\right)^{\frac{1}{\tau}} \max _{T^{\prime} \in \mathcal{T}^{\prime}}\left\|\nabla\left(f-\mathrm{I}_{T^{\prime}}^{m-1} f\right)\right\|_{L^{p}\left(T^{\prime}\right)} \\
& \leq\left(C_{1}+1\right)\left(C_{0}^{3} /\left|T_{\mathrm{eq}}\right|\right)^{\frac{1}{\tau}}\left(\int_{\Omega}(\operatorname{det} \mathcal{M})^{\frac{m-1}{4} \tau} d z\right)^{\frac{1}{\tau}}, \\
& \leq C_{2}\left\|L_{m}\left(d^{m} f / m !\right)\right\|_{L^{\tau}(\Omega)},
\end{aligned}
$$

where $C_{2}=\left(C_{1}+1\right)\left(C_{0}^{3} /\left|T_{\text {eq }}\right|\right)^{\frac{1}{\tau}} C_{L}$. We used the definition $(7)$ of the exponent $\tau$ in the first line, (36) in the second line and (33) in the last. As announced the triangulation $\mathcal{T}^{\prime}$ satisfies the optimal error estimate of Theorem 1, up to the multiplicative constant $C_{2}$.

\section{Study of the shape function}

This section is devoted to the close study of the shape function $L_{m, p}$, using algebraic techniques. Our approach is based on the variant $L_{m}$ introduced in (32), defined for all $\pi \in \mathbb{H}_{m}$ by

$$
L_{m}(\pi):=\inf \left\{(\operatorname{det} M)^{\frac{m-1}{4}} ; M \in S_{2}^{+} \text {s.t. }\left\|(\nabla \pi) \circ M^{-\frac{1}{2}}\right\| \leq 1\right\},
$$

and which is uniformly equivalent to $L_{m, p}$ according to Lemma 1.6 .

\subsection{Explicit minimizers}

We describe the solution to the optimization problem appearing in (37) when $m \in\{2,3\}$. The case $m=2$, which corresponds to piecewise linear finite elements is already known, and discussed in detail in [16, 3] and [14] for functions of more than two variables. In contrast the results in the case $m=3$ are entirely new, although this case, which corresponds to piecewise quadratic elements, has already been discussed in 10 .

In order to present our results, we introduce some notation. For any homogeneous quadratic polynomial $\pi \in \mathbb{H}_{2}, \pi=a x^{2}+2 b x y+c y^{2}$, we define the symmetric matrix

$$
[\pi]=\left(\begin{array}{cc}
a & b \\
b & c
\end{array}\right) .
$$

For all $\pi \in \mathbb{H}_{2}, \pi=a x^{2}+2 b x y+c y^{2}$, we define

$$
\mathcal{M}_{2}(\pi):=4[\pi]^{2}=4\left(\begin{array}{cc}
a & b \\
b & c
\end{array}\right)^{2}=4\left(\begin{array}{cc}
a^{2}+b^{2} & a b+b c \\
a b+b c & b^{2}+c^{2}
\end{array}\right) .
$$


For all $\pi \in \mathbb{H}_{3}, \pi=a x^{3}+3 b x^{2} y+3 c x y^{2}+d y^{3}$, we define

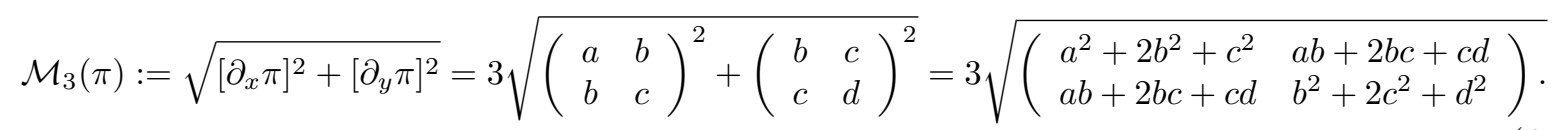

We say that a polynomial $\pi \in \mathbb{H}_{m}$ is univariate if there exists $\lambda, \alpha, \beta \in \mathbb{R}$ such that $\pi=\lambda(\alpha x+\beta y)^{m}$.

Proposition 2.1. $\quad$. If $\pi \in \mathbb{H}_{2}$ is not univariate, then the matrix $\mathcal{M}_{2}(\pi)$ is the unique minimizer of the optimization problem appearing in (32).

ii. The map $\pi \in \mathbb{H}_{3} \rightarrow \mathcal{M}_{3}(\pi)$ is a near-minimizer of the problem (32) in the following sense. If $\pi \in \mathbb{H}_{3}$ is not univariate, then $\mathcal{M}_{3}(\pi)$ is non-degenerate and $\left\|(\nabla \pi) \circ \mathcal{M}_{3}(\pi)^{-\frac{1}{2}}\right\| \leq \sqrt{2}$ (hence $\left.\left\|(\nabla \pi) \circ\left(\sqrt{2} \mathcal{M}_{3}(\pi)\right)^{-\frac{1}{2}}\right\| \leq 1\right)$. Furthermore there exists a constant $C$, independent of $\pi$, such that

$$
\sqrt{\operatorname{det} \mathcal{M}_{3}(\pi)} \leq C L_{3}(\pi) .
$$

Proof. According to $\sqrt{29},\left\|(\nabla \pi) \circ M^{-\frac{1}{2}}\right\| \leq K$ is equivalent to $|\nabla \pi(z)| \leq K|z|_{M}^{m-1}$ for all $z \in \mathbb{R}^{2}$, where $\pi \in \mathbb{H}_{m}, M \in S_{2}^{+}$and $K>0$ are arbitrary.

We first consider an homogeneous polynomial $\pi \in \mathbb{H}_{2}$, which is not univariate. For all $z \in \mathbb{R}^{2}$ one has $\nabla \pi(z)=2[\pi] z$, and therefore $|\nabla \pi(z)|^{2}=z^{\mathrm{T}} \mathcal{M}_{2}(\pi) z$. On the other hand consider $M \in S_{2}^{+}$such that $|\nabla \pi(z)|^{2}=z^{\mathrm{T}} \mathcal{M}_{2}(\pi) z \leq z^{\mathrm{T}} M z$. We thus have $\mathcal{M}_{2}(\pi) \leq M$ in the sense of symmetric matrices, which implies that $\operatorname{det} \mathcal{M}_{2}(\pi) \leq \operatorname{det} M$ with equality if and only if $\mathcal{M}_{2}(\pi)=M$, since $\mathcal{M}_{2}(\pi)$ is positive definite. This concludes the proof of the first point.

We now consider $\pi \in \mathbb{H}_{3}$, which is again not univariate. In the sense of symmetric matrices, we have

$$
\mathcal{M}_{3}(\pi)=\sqrt{\left[\partial_{x} \pi\right]^{2}+\left[\partial_{y} \pi\right]^{2}} \geq \sqrt{\left[\partial_{x} \pi\right]^{2}}=\left|\left[\partial_{x} \pi\right]\right|,
$$

where we used the fact that the square root $\sqrt{ } \cdot: S_{2}^{\oplus} \rightarrow S_{2}^{\oplus}$ is increasing. It follows that

$$
|\nabla \pi(z)|^{2}=\left|\partial_{x} \pi(z)\right|^{2}+\left|\partial_{y} \pi(z)\right|^{2} \leq 2\left(z^{\mathrm{T}} \mathcal{M}_{3}(\pi) z\right)^{2},
$$

hence $\mathcal{M}_{3}(\pi)$ satisfies the constraint $\left\|(\nabla \pi) \circ \mathcal{M}_{3}(\pi)^{-\frac{1}{2}}\right\| \leq \sqrt{2}$. Note that

$$
\operatorname{det} \mathcal{M}_{3}(\pi)=9 \sqrt{\left(a^{2}+2 b^{2}+c^{2}\right)\left(b^{2}+2 c^{2}+d^{2}\right)-(a b+2 b c+c d)^{2}} .
$$

We postpone the proof of (41) to $\$ 2.2$ right after $(48)$, as we develop a general method for obtaining simple equivalents of the functions $L_{m}$.

Remark 2.2. It was proposed in [3, 14] to generate an anisotropic mesh $\mathcal{T}$ of a domain $\Omega$ via the transport of a uniform mesh $\mathcal{T}^{\prime}$ of an auxiliary domain $\Omega^{\prime}$ by a diffeomorphism $F: \Omega \rightarrow \Omega^{\prime}$. Without entering the details of this approach, we may describe one of its successes. Assume that one wishes to approximate a strongly convex function $f \in C^{2}(\bar{\Omega})$, using linear finite elements. Define $F(z):=2 \nabla f(z)$ and $\Omega^{\prime}:=F(\Omega) \subset \mathbb{R}^{2}$. Consider a uniform mesh $\mathcal{T}^{\prime}$ of $\Omega^{\prime}$, of mesh size $h>0$, and denote by $\mathcal{T}$ the collection of triangles obtained as follows: for each triangle $T^{\prime} \in \mathcal{T}^{\prime}$, of vertices $v_{1}, v_{2}, v_{3}$, the set $\mathcal{T}$ contains the triangle $T$ of vertices $F^{-1}\left(v_{1}\right), F^{-1}\left(v_{2}\right), F^{-1}\left(v_{3}\right)$. If the parameter $h>0$ is sufficiently small, then $\mathcal{T}$ is a triangulation since $F: \Omega \rightarrow \Omega^{\prime}$ is a diffeomorphism. Furthermore we obtain using (14) that $\mathcal{T}$ is adapted in the sense of (35) to the metric $H: \Omega \rightarrow S_{2}^{+}$defined by

$$
H(z):=h^{-2} d F(z)^{\mathrm{T}} d F(z)=h^{-2} 4\left(d^{2} f(z)\right)^{2}=h^{-2} \mathcal{M}_{2}\left(\pi_{z}\right),
$$

hence the optimal metric, see (34), for the approximation $f$ in the $W^{1, \infty}$ semi-norm! (The control of the measure of sliverness of the elements of $\mathcal{T}$ remains, however, an open problem with this method.)

Let us finally mention that, although they are derived from the coefficients of $\pi$, the maps $\pi \mapsto \mathcal{M}_{m}(\pi)$ for $m \in\{2,3\}$ are invariant under rotation, and therefore not tied to the chosen system of coordinate $(x, y)$, as expressed by the following result. 
Proposition 2.3. For any $m \in\{2,3\}$, any $\pi \in \mathbb{H}_{m}$ and any unitary matrix $U \in \mathcal{O}_{2}$, one has

$$
\mathcal{M}_{m}(\pi \circ U)=U^{\mathrm{T}} \mathcal{M}_{m}(\pi) U
$$

Proof. Let $\pi \in \mathbb{H}_{2}$ and let $U \in \mathcal{O}_{2}$. Then, as announced,

$$
\mathcal{M}_{2}(\pi \circ U)=4[\pi \circ U]^{2}=4\left(U^{\mathrm{T}}[\pi] U\right)^{2}=U^{\mathrm{T}} \mathcal{M}_{2}(\pi) U
$$

Let $\pi \in \mathbb{H}_{3}$ and denote by $\left(u_{i j}\right)_{1 \leq i, j \leq 2}$ the entries of the unitary matrix $U$. Then

$$
\left[\partial_{x}(\pi \circ U)\right]=u_{11} U^{\mathrm{T}}\left[\partial_{x} \pi\right] U+u_{12} U^{\mathrm{T}}\left[\partial_{y} \pi\right] U \quad \text { and } \quad\left[\partial_{y}(\pi \circ U)\right]=u_{21} U^{\mathrm{T}}\left[\partial_{x} \pi\right] U+u_{22} U^{\mathrm{T}}\left[\partial_{y} \pi\right] U
$$

Hence, since $U$ is unitary,

$$
\begin{aligned}
& {\left[\partial_{x}(\pi \circ U)\right]^{2}+\left[\partial_{y}(\pi \circ U)\right]^{2}} \\
& =\left(u_{11}^{2}+u_{21}^{2}\right) U^{\mathrm{T}}\left[\partial_{x} \pi\right]^{2} U+\left(u_{11} u_{12}+u_{21} u_{22}\right) U^{\mathrm{T}}\left(\left[\partial_{x} \pi\right]\left[\partial_{y} \pi\right]+\left[\partial_{y} \pi\right]\left[\partial_{x} \pi\right]\right) U+\left(u_{12}^{2}+u_{22}^{2}\right) U^{\mathrm{T}}\left[\partial_{y} \pi\right]^{2} U \\
& =U^{\mathrm{T}}\left[\partial_{x} \pi\right]^{2} U+U^{\mathrm{T}}\left[\partial_{y} \pi\right]^{2} U
\end{aligned}
$$

Therefore

$$
\mathcal{M}_{3}(\pi \circ U)=\sqrt{U^{\mathrm{T}}\left[\partial_{x} \pi\right]^{2} U+U^{\mathrm{T}}\left[\partial_{y} \pi\right]^{2} U}=U^{\mathrm{T}} \sqrt{\left[\partial_{x} \pi\right]^{2}+\left[\partial_{y} \pi\right]^{2}} U=U^{\mathrm{T}} \mathcal{M}_{3}(\pi) U
$$

which concludes the proof.

\subsection{Polynomial equivalents}

We introduce equivalents of the shape function $\pi \mapsto L_{m}(\pi)$ on $\mathbb{H}_{m}$, which can be written in analytic form in terms of the coefficients of $\pi \in \mathbb{H}_{m}$. As a starter we infer from Point i of Proposition 2.1 that for any $\pi \in \mathbb{H}_{2}$

$$
L_{2}(\pi)=\left(\operatorname{det} \mathcal{M}_{2}(\pi)\right)^{\frac{1}{4}}=2 \sqrt{|\operatorname{det}[\pi]|} .
$$

For each $r \geq 2$ we denote by $\mathbb{H}_{r}$ the space of homogeneous bivariate polynomials of degree $r$, as in (4) for $r=m$, equipped with the norm

$$
\|\mu\|:=\sup _{z \neq 0} \frac{|\mu(z)|}{|z|^{r}}
$$

For $\mu \in \mathbb{H}_{r}$ and $A \in \mathrm{M}_{2}$, we define the homogeneous polynomial $\mu \circ A \in \mathbb{H}_{r}$ by $\mu \circ A(z):=\mu(A z)$, $z \in \mathbb{R}^{2}$. Observe that for $M \in S_{2}^{+}$one has

$$
\left\|\mu \circ M^{-\frac{1}{2}}\right\|=\sup _{z \neq 0} \frac{|\mu(z)|}{|z|_{M}^{r}} .
$$

We now introduce a variant $K_{r}:=\mathbb{H}_{r} \rightarrow \mathbb{R}_{+}$of the shape function $L_{m}$, which was first defined in [8], and later studied in [18, in the context of optimal mesh adaptation for finite element approximation in the $L^{p}$ norm. (More precisely, due to different conventions, the function $K_{r}$ is tied to the function $K_{r}^{\mathcal{E}}$ defined in [18] by the equality $K_{r}=\boldsymbol{\pi}^{-\frac{r}{2}} K_{r}^{\mathcal{E}}$.) For each $\mu \in \mathbb{H}_{r}$ we define

$$
K_{r}(\mu):=\inf \left\{(\operatorname{det} M)^{\frac{r}{4}} ; M \in S_{2}^{+} \text {s.t. }\left\|\mu \circ M^{-\frac{1}{2}}\right\| \leq 1\right\} .
$$

Observe that $|\nabla \pi|^{2}:=\left(\partial_{x} \pi\right)^{2}+\left(\partial_{y} \pi\right)^{2} \in \mathbb{H}_{2 m-2}$ for each $\pi \in \mathbb{H}_{m}$, and that clearly

$$
L_{m}(\pi)=\sqrt{K_{2 m-2}\left(|\nabla \pi|^{2}\right)} .
$$

Given a pair of non negative functions $Q$ and $R$ on $\mathbb{H}_{m}$, we write $Q \sim R$ if and only if there exists a constant $C \geq 1$ such that $C^{-1} Q \leq R \leq C Q$ holds uniformly on $\mathbb{H}_{m}$. We sometimes slightly abuse 
notations and write $Q(\pi) \sim R(\pi)$. We say that a function $Q$ is a polynomial on $\mathbb{H}_{m}$ if there exists a polynomial $P$ of $m+1$ real variables such that for all $a_{0}, \cdots, a_{m} \in \mathbb{R}$,

$$
Q\left(\sum_{i=0}^{m} a_{i} x^{i} y^{m-i}\right)=P\left(a_{0}, \cdots, a_{m}\right) .
$$

We define $\operatorname{deg} Q:=\operatorname{deg} P$, and we say that $Q$ is homogeneous if $P$ is homogeneous.

The following equivalences were established in [18] : for $\pi \in \mathbb{H}_{2}$

$$
K_{2}(\pi) \sim \sqrt{|\operatorname{det}[\pi]|},
$$

and for $\pi \in \mathbb{H}_{3}$

$$
K_{3}(\pi) \sim \sqrt[4]{|\operatorname{disc}(\pi)|},
$$

where $\operatorname{disc}(\pi)$ denotes the discriminant of the cubic polynomial $\pi$, which is defined by

$$
\operatorname{disc}\left(a x^{3}+3 b x^{2} y+3 c x y^{2}+d y^{3}\right)=4\left(a b-c^{2}\right)\left(b c-d^{2}\right)-(a d-b c)^{2} .
$$

More generally for each $r \geq 2$ an explicit homogenous polynomial $Q$ on $\mathbb{H}_{r}$ is introduced in [18, which satisfies

$$
K_{r} \sim \sqrt[d]{|Q|}, \text { with } d:=\operatorname{deg} Q .
$$

Combining this result with (44) we obtain an explicit equivalent of the functions $L_{m}$.

Proposition 2.4. Let $m \geq 2$ and let $Q$ be an homogeneous polynomial on $\mathbb{H}_{2 m-2}$ such that $K_{2 m-2} \sim$ $\sqrt[d]{|Q|}$, where $d=\operatorname{deg} Q$. Let $Q_{*}$ be the polynomial on $\mathbb{H}_{m}$ defined by

$$
Q_{*}(\pi):=Q\left(|\nabla \pi|^{2}\right),
$$

then $L_{m} \sim \sqrt[2 d]{Q_{*}}$ on $\mathbb{I H}_{m}$.

This construction uses an equivalent $\sqrt[d]{|Q|}$ of $K_{2 m-2}$ to produce an equivalent of $L_{m}$. Unfortunately, as $m$ increases, the practical construction of $Q$ becomes more involved and the degree $d$ quickly raises. In the following theorem, we build an equivalent to $L_{m}$ from an equivalent of $K_{m-1}$ instead of $K_{2 m-2}$, which is therefore simpler.

Theorem 2.5. Let $m \geq 3$ and let $Q$ be an homogeneous polynomial on $\mathbb{H}_{m-1}$ such that $K_{m-1} \sim \sqrt[d]{|Q|}$, where $d=\operatorname{deg} Q$. Let $\left(Q_{k}\right)_{0 \leq k \leq r}$ be the homogeneous polynomials of degree $d$ on $\mathbb{H}_{m-1} \times \mathbb{H}_{m-1}$ such that for all $u, v \in \mathbb{R}$ and all $\pi_{1}, \pi_{2} \in \mathbb{H}_{m}$ one has

$$
Q\left(u \pi_{1}+v \pi_{2}\right)=\sum_{0 \leq k \leq d}\left(\begin{array}{l}
d \\
k
\end{array}\right) u^{k} v^{d-k} Q_{k}\left(\pi_{1}, \pi_{2}\right),
$$

where $\left(\begin{array}{l}d \\ k\end{array}\right):=\frac{d !}{k !(d-k) !}$. Let $Q_{*}$ be the polynomial defined for all $\pi \in \mathbb{H}_{m}$ by

$$
Q_{*}(\pi):=\sum_{0 \leq k \leq d}\left(\begin{array}{l}
d \\
k
\end{array}\right) Q_{k}\left(\partial_{x} \pi, \partial_{y} \pi\right)^{2}
$$

then $L_{m} \sim \sqrt[2 d]{Q_{*}}$ on $\mathbb{I H}_{m}$.

Proof. See Appendix B.

Using this construction and (45) we obtain an equivalent of $L_{3}$ as follows. Let $\pi_{1}=a x^{2}+2 b x y+c y^{2}$ and $\pi_{2}=a^{\prime} x^{2}+2 b^{\prime} x y+c^{\prime} y^{2}$ be two elements of $\mathbb{H}_{2}$. We obtain

$$
\begin{aligned}
\operatorname{det}\left(\left[u \pi_{1}+v \pi_{2}\right]\right) & =\left(u a+v a^{\prime}\right)\left(u c+v c^{\prime}\right)-\left(u b+v b^{\prime}\right)^{2} \\
& =u^{2}\left(a c-b^{2}\right)+u v\left(a c^{\prime}+a^{\prime} c-2 b b^{\prime}\right)+v^{2}\left(a^{\prime} c^{\prime}-b^{\prime 2}\right) .
\end{aligned}
$$


Applying the construction of Theorem 2.5 to $\pi=a x^{3}+3 b x^{2} y+3 c x y^{2}+d y^{3} \in \mathbb{H}_{3}$ we obtain

$$
L_{3}(\pi) \sim 3 \sqrt[4]{\left(a c-b^{2}\right)^{2}+(a d-b c)^{2} / 2+\left(b d-c^{2}\right)^{2}} .
$$

Remarking that

$$
2\left[\left(a c-b^{2}\right)^{2}+(a d-b c)^{2} / 2+\left(b d-c^{2}\right)^{2}\right]=\left(a^{2}+2 b^{2}+c^{2}\right)\left(b^{2}+2 c^{2}+d^{2}\right)-(a b+2 b c+c d)^{2},
$$

and using equation $\sqrt{42}$ we obtain that $L_{3}(\pi) \sim \sqrt{\operatorname{det} \mathcal{M}_{3}(\pi)}$. This point concludes the proof of Proposition 2.1 and thus establishes that the map $\mathcal{M}_{3}$ defined in 40 can be used for optimal mesh adaptation for quadratic finite elements.

Using (46) we similarly obtain an equivalent of $L_{4}$. Denoting $\pi=a x^{4}+4 b x^{3} y+6 c x^{2} y^{2}+4 d x y^{3}+e y^{4}$ :

$$
\begin{aligned}
L_{4}(\pi)^{8} \sim & \left(3 b^{2} c^{2}-4 a c^{3}-4 b^{3} d+6 a b c d-a^{2} d^{2}\right)^{2} \\
& +\left(2 b c^{3}-6 a c^{2} d+4 a b d^{2}-4 b^{3} e+6 a b c e-2 a^{2} d e\right)^{2} / 4 \\
& +\left(3 c^{4}-6 b c^{2} d+8 b^{2} d^{2}-6 a c d^{2}-6 b^{2} c e+6 a c^{2} e+2 a b d e-a^{2} e^{2}\right)^{2} / 6 \\
& +\left(2 c^{3} d-4 a d^{3}-6 b c^{2} e+4 b^{2} d e+6 a c d e-2 a b e^{2}\right)^{2} / 4 \\
& +\left(3 c^{2} d^{2}-4 b d^{3}-4 c^{3} e+6 b c d e-b^{2} e^{2}\right)^{2} .
\end{aligned}
$$

The following proposition identifies the polynomials $\pi \in \mathbb{H}_{m}$ for which $L_{m}(\pi)=0$, and therefore the values of $d^{m} f$ for which anisotropic mesh adaptation may lead to super-convergence.

Proposition 2.6. Let $m \geq 2$ and let $t_{m}:=\left\lfloor\frac{m+3}{2}\right\rfloor$. Then for all $\pi \in \mathbb{H}_{m}$,

$$
L_{m}(\pi)=0 \text { if and only if } \pi=(\alpha x+\beta y)^{t_{m}} \tilde{\pi} \text { for some } \alpha, \beta \in \mathbb{R} \text { and } \tilde{\pi} \in \mathbb{H}_{m-t_{m}} \text {. }
$$

Proof. It was established in 18 that $K_{2 m-2}\left(\pi_{*}\right)=0$ if and only if $\pi_{*} \in \mathbb{H}_{2 m-2}$ has a linear factor of multiplicity $m$. We therefore obtain, using (44), that $L_{m}(\pi)=0$ if and only $|\nabla \pi|^{2}$ is a multiple of $l^{m}$, where $l$ is of the form $l=\alpha x+\beta y$.

Let us first assume that $|\nabla \pi|^{2}=\left(\partial_{x} \pi\right)^{2}+\left(\partial_{y} \pi\right)^{2}$ has such a form. Clearly $\left(\partial_{x} \pi\right)^{2}$ and $\left(\partial_{y} \pi\right)^{2}$ are both multiples of $l^{m}$. Therefore $\partial_{x} \pi$ and $\partial_{y} \pi$ are multiples of $l^{s}$ where $s$ is an integer such that $2 s \geq m$, hence $s \geq t_{m}-1$. We therefore have

$$
\partial_{x} \pi=l^{s} \pi_{1} \text { and } \partial_{y} \pi=l^{s} \pi_{2} \text { where } \pi_{1}, \pi_{2} \in \mathbb{H}_{m-1-s} .
$$

Recalling that $l=\alpha x+\beta y$ we obtain

$$
0=\partial_{y x}^{2} \pi-\partial_{x y}^{2} \pi=l^{s}\left(\partial_{y} \pi_{1}-\partial_{x} \pi_{2}\right)+s l^{s-1}\left(\beta \pi_{1}-\alpha \pi_{2}\right),
$$

hence $\beta \pi_{1}-\alpha \pi_{2}$ is a multiple of $l$. Since $\pi$ is homogenous of degree $m$ it obeys the Euler identity $m \pi(z)=\langle z, \nabla \pi(z)\rangle$ for all $z=(x, y) \in \mathbb{R}^{2}$. Assuming without loss of generality that $\alpha \neq 0$, we therefore obtain

$$
m \pi(x, y)=l^{s}\left(x \pi_{1}+y \pi_{2}\right)=l^{s}\left((\alpha x+\beta y) \frac{\pi_{1}}{\alpha}+\frac{y}{\alpha}\left(\alpha \pi_{2}-\beta \pi_{1}\right)\right)
$$

which shows that $\pi$ is a multiple of $l^{s+1}$, hence of $l^{t_{m}}$.

Conversely if $\pi$ is a multiple of $l^{t_{m}}$ then $\partial_{x} \pi$ and $\partial_{y} \pi$ are both multiples of $l^{t_{m}-1}$. Since $2\left(t_{m}-1\right) \geq m$ the polynomial $|\nabla \pi|^{2}$ is a multiple of $l^{m}$ which concludes the proof.

\section{Proof of the main result}

This section is devoted to the proof of Theorem 2. We thus consider a fixed bounded polygonal domain $\Omega \subset \mathbb{R}^{2}$, an integer $m \geq 2$, an exponent $1 \leq p<\infty$ and a function $f \in C^{m}(\bar{\Omega})$. 
The Taylor development of $f$ close to a point $z \in \Omega$ is given by two polynomials $\mu_{z} \in \mathbb{P}_{m-1}$ and $\pi_{z} \in \mathbb{H}_{m}: f(z+h)=\mu_{z}(h)+\pi_{z}(h)+o\left(|h|^{m}\right)$, where $h \in \mathbb{R}^{2}$ is small. The Taylor development of the function $\nabla f: \Omega \rightarrow \mathbb{R}^{2}$ close to a point $z \in \Omega$ is obtained by derivation of the previous one:

$$
\nabla f(z+h)=\nabla \mu_{z}(h)+\nabla \pi_{z}(h)+o\left(|h|^{m-1}\right),
$$

and the corresponding Taylor formula reads as follows: for any $z, h$ such that $[z, z+h] \subset \Omega$

$$
\nabla f(z+h)=\nabla \mu_{z}(h)+(m-1) \int_{t=0}^{1} \nabla \pi_{z+t h}(h)(1-t)^{m-2} d t .
$$

We denote by $\omega$ the modulus of continuity of the function $z \in \Omega \mapsto \nabla \pi_{z} \in \mathbb{H}_{m-1}^{2}$ : for each $r>0$

$$
\omega(r):=\sup \left\{\left\|\nabla \pi_{z}-\nabla \pi_{z^{\prime}}\right\| ; z, z^{\prime} \in \Omega \text { s.t. }\left|z-z^{\prime}\right| \leq r\right\},
$$

where we recall that $\|\mu\|=\sup \left\{|\mu(z)| /|z|^{m-1} ; z \neq 0\right\}$ for all $\mu \in \mathbb{H}_{m-1}^{2}$, see 28 .

Our first lemma is an estimation on a single triangle of the gradient interpolation error of $f$.

Lemma 3.1. There exists a constant $C_{\star}=C_{\star}(f, m)$ such that the following holds.

i. For any triangle $T \subset \bar{\Omega}$, and any $z \in T$ one has

$$
\left|\left\|\nabla\left(f-\mathrm{I}_{T}^{m-1} f\right)\right\|_{L^{p}(T)}-\left\|\nabla\left(\pi_{z}-\mathrm{I}_{T}^{m-1} \pi_{z}\right)\right\|_{L^{p}(T)}\right| \leq C_{\star} \omega(\operatorname{diam}(T))|T|^{\frac{1}{\tau}} \rho(T)^{\frac{m+1}{2}}
$$

ii. For any triangle $T \subset \bar{\Omega}$ one has

$$
\left\|\nabla\left(f-\mathrm{I}_{T}^{m-1} f\right)\right\|_{L^{p}(T)} \leq C_{\star}|T|^{\frac{1}{p}} \operatorname{diam}(T)^{m-1} S(T) .
$$

Proof. The point $z$ is fixed throughout this proof. We define a function $g \in C^{m}(\bar{\Omega})$ by the equality

$$
g(z+h):=f(z+h)-\mu_{z}(h)-\pi_{z}(h),
$$

for all $h$ such that $z+h \in \bar{\Omega}$. We have

$$
\begin{aligned}
\left|\left\|\nabla\left(f-\mathrm{I}_{T}^{m-1} f\right)\right\|_{L^{p}(T)}-\left\|\nabla\left(\pi_{z}-\mathrm{I}_{T}^{m-1} \pi_{z}\right)\right\|_{L^{p}(T)}\right| & \leq\left\|\nabla\left(\left(f-\pi_{z}\right)-\mathrm{I}_{T}^{m-1}\left(f-\pi_{z}\right)\right)\right\|_{L^{p}(T)} \\
& =\left\|\nabla\left(g-\mathrm{I}_{T}^{m-1} g\right)\right\|_{L^{p}(T)} \\
& \leq|T|^{\frac{1}{p}}\left\|\nabla\left(g-\mathrm{I}_{T}^{m-1} g\right)\right\|_{L^{\infty}(T)} \\
& \leq C \rho(T)|T|^{\frac{1}{p}}\|\nabla g\|_{L^{\infty}(T)},
\end{aligned}
$$

where we used the reverse triangle inequality in the first line, the translation invariance $(8)$ and the equality $\mu_{z}=\mathrm{I}_{T}^{m-1} \mu_{z}$ in the second line, Jensen's inequality in the third line, and Lemma 1.3 in the last line combined with the inequality $1 \leq S(T) \leq \rho(T)$, see 21$)$. We have

$$
\nabla g(z+h)=\nabla f(z+h)-\nabla \mu_{z}(h)-\nabla \pi_{z}(h)=(m-1) \int_{t=0}^{1}\left(\nabla \pi_{z+t h}(h)-\nabla \pi_{z}(h)\right)(1-t)^{m-2} d t,
$$

therefore $\|\nabla g(z+h)\| \leq|h|^{m-1} \omega(h)$. Furthermore if $z \in T$ and $z+h \in T$, then $|h|^{2} \leq \operatorname{diam}(T)^{2} \leq$ $\left(4 /\left|T_{\text {eq }}\right|\right)|T| \rho(T)$ according to Lemma 1.1 . This concludes the proof of Point i, provided that $C_{\star} \geq$ $C\left(4 /\left|T_{\text {eq }}\right|\right)^{\frac{m-1}{2}}$.

We now turn to the proof of Point ii, and for that purpose we consider a fixed point $z \in T$. Changing our previous notation we denote by $g \in C^{m}(\bar{\Omega})$ the function defined by $g(z+h):=f(z+h)-\mu_{z}(h)$ for all $h$ such that $z+h \in \bar{\Omega}$. We obtain

$$
\left\|\nabla\left(f-\mathrm{I}_{T}^{m-1} f\right)\right\|_{L^{p}(T)}=\left\|\nabla\left(g-\mathrm{I}_{T}^{m-1} g\right)\right\|_{L^{p}(T)} \leq|T|^{\frac{1}{p}}\left\|\nabla\left(g-\mathrm{I}_{T}^{m-1} g\right)\right\|_{L^{\infty}(T)} \leq C|T|^{\frac{1}{p}}\|\nabla g\|_{L^{\infty}(T)},
$$

where we used successively that the interpolation operator reproduces the elements of $\mathbb{P}_{m-1}$, Jensen's inequality, and Lemma 1.3 . On the other hand we obtain using (50).

$$
\nabla g(z+h)=\nabla f(z+h)-\nabla \mu_{z}(h)=(m-1) \int_{t=0}^{1} \nabla \pi_{z+t h}(h)(1-t)^{m-2} d t,
$$

hence $|\nabla g(z+h)| \leq|h|^{m-1} \sup \left\{\left\|\nabla \pi_{z}\right\| ; z \in \Omega\right\}$. If $z \in T$ and $z+h \in T$, then $|h| \leq \operatorname{diam}(T)$, which concludes the proof of 53 provided that $C_{\star} \geq C \sup \left\{\left\|\nabla \pi_{z}\right\| ; z \in \Omega\right\}$. 


\subsection{The lower error estimate $(12)$}

Under the hypotheses of Lemma 3.1 and recalling from (8) that $\left\|\nabla\left(\pi-\mathrm{I}_{T}^{m-1} \pi\right)\right\|_{L^{p}(T)} \geq|T|^{\frac{1}{\tau}} L_{m, p}(\pi)$, we obtain

$$
\left\|\nabla\left(f-\mathrm{I}_{T}^{m-1} f\right)\right\|_{L^{p}(T)} \geq|T|^{\frac{1}{\tau}}\left(L_{m, p}\left(\pi_{z}\right)-C_{\star} \omega(\operatorname{diam} T) \rho(T)^{\frac{m+1}{2}}\right) .
$$

We consider an admissible sequence of triangulations $\left(\mathcal{T}_{N}\right)_{N \geq N_{0}}$. For all $N \geq N_{0}$, all $T \in \mathcal{T}_{N}$ and all $z \in T$, we define $\phi_{N}(z):=|T|$ and

$$
\psi_{N}(z):=\left(L_{m, p}\left(\pi_{z}\right)-C_{\star} \omega(\operatorname{diam}(T)) \rho(T)^{\frac{m+1}{2}}\right)_{+},
$$

where $\lambda_{+}:=\max \{\lambda, 0\}$. Holder's inequality $\int f_{1} f_{2} \leq\left\|f_{1}\right\|_{p_{1}}\left\|f_{2}\right\|_{p_{2}}$, applied to the functions $f_{1}=$ $\phi_{N}^{\frac{(m-1) \tau}{2}} \psi_{N}^{\tau}$ and $f_{2}=\phi_{N}^{-\frac{(m-1) \tau}{2}}$, and the exponents $p_{1}=\frac{p}{\tau}$ and $p_{2}=\frac{2}{(m-1) \tau}$, yields

$$
\int_{\Omega} \psi_{N}^{\tau} \leq\left(\int_{\Omega} \phi_{N}^{\frac{(m-1) p}{2}} \psi_{N}^{p}\right)^{\frac{\tau}{p}}\left(\int_{\Omega} \phi_{N}^{-1}\right)^{\frac{(m-1) \tau}{2}} .
$$

Furthermore for any $T \in \mathcal{T}_{N}$ and any $z \in T$, we obtain using (54)

$$
\phi_{N}(z)^{\frac{(m-1) p}{2}} \psi_{N}(z)^{p}=|T|^{\frac{p}{\tau}-1} \psi_{N}(z)^{p} \leq \frac{1}{|T|}\left\|\nabla\left(f-\mathrm{I}_{T}^{m-1} f\right)\right\|_{L^{p}(T)}^{p},
$$

hence

$$
\int_{\Omega} \phi_{N}^{\frac{(m-1) p}{2}} \psi_{N}^{p} \leq \sum_{T \in \mathcal{T}_{N}} \frac{1}{|T|} \int_{T}\left\|\nabla\left(f-\mathrm{I}_{T}^{m-1} f\right)\right\|_{L^{p}(T)}^{p}=\left\|\nabla\left(f-\mathrm{I}_{\mathcal{T}_{N}}^{m-1} f\right)\right\|_{L^{p}(\Omega)}^{p} .
$$

Elevating 55 to the power $\frac{1}{\tau}$, injecting the above estimate and observing that $\int_{\Omega} \phi_{N}^{-1}=\# \mathcal{T}_{N} \leq N$, we thus obtain

$$
\left\|\psi_{N}\right\|_{L^{\tau}(\Omega)} \leq\left\|\nabla\left(f-\mathrm{I}_{\mathcal{T}_{N}}^{m-1} f\right)\right\|_{L^{p}(\Omega)} N^{\frac{m-1}{2}} .
$$

Since the sequence $\left(\mathcal{T}_{N}\right)_{N \geq N_{0}}$ is admissible, there exists a constant $C_{A}>0$ such that for all $N$ and all $T \in \mathcal{T}_{N}$ we have $\operatorname{diam}(T) \leq C_{A} N^{-\frac{1}{2}}$. We introduce a subset of $\mathcal{T}_{N}^{\prime} \subset \mathcal{T}_{N}$ which gathers the most degenerate triangles

$$
\mathcal{T}_{N}^{\prime}=\left\{T \in \mathcal{T}_{N} ; \rho(T) \geq \omega\left(C_{A} N^{-\frac{1}{2}}\right)^{\frac{-1}{m+1}}\right\}
$$

where $\omega$ is defined by (51). We denote by $\Omega_{N}^{\prime}$ the portion of $\Omega$ covered by $\mathcal{T}_{N}^{\prime}$. For all $z \in \Omega \backslash \Omega_{N}^{\prime}$ one has

$$
\psi_{N}(z) \geq L_{m, p}\left(\pi_{z}\right)-C_{\star} \sqrt{\omega\left(C_{A} N^{-\frac{1}{2}}\right)} .
$$

Hence, with the convention $L_{m, p}\left(d^{m} f(z) / m !\right):=L_{m, p}\left(\pi_{z}\right)$,

$$
\begin{aligned}
\left\|\psi_{N}\right\|_{L^{\tau}(\Omega)}^{\tau} & \geq\left\|\left(L_{m, p}\left(\frac{d^{m} f}{m !}\right)-C_{\star} \sqrt{\omega\left(C_{A} N^{-\frac{1}{2}}\right)}\right)_{+}\right\|_{L^{\tau}\left(\Omega \backslash \Omega_{N}^{\prime}\right)}^{\tau} \\
& \geq\left\|\left(L_{m, p}\left(\frac{d^{m} f}{m !}\right)-C_{\star} \sqrt{\omega\left(C_{A} N^{-\frac{1}{2}}\right)}\right)_{+}\right\|_{L^{\tau}(\Omega)}^{\tau}-C^{\tau}\left|\Omega_{N}^{\prime}\right|,
\end{aligned}
$$

where $C:=\max \left\{L_{m, p}\left(\pi_{z}\right) ; z \in \Omega\right\}<\infty$. We next observe that $\left|\Omega_{N}^{\prime}\right| \rightarrow 0$ as $N \rightarrow+\infty$ : indeed for all $T \in \mathcal{T}_{N}^{\prime}$ we obtain using Lemma 1.1

$$
|T| \leq\left|T_{\mathrm{eq}}\right| \operatorname{diam}(T)^{2} \rho(T)^{-1} \leq\left|T_{\mathrm{eq}}\right| C_{A}^{2} N^{-1} \omega\left(C_{A} N^{-\frac{1}{2}}\right)^{\frac{1}{m+1}}
$$

Since $\#\left(\mathcal{T}_{N}^{\prime}\right) \leq N$, we obtain $\left|\Omega_{N}^{\prime}\right| \leq\left|T_{\text {eq }}\right| C_{A}^{2} \omega\left(C_{A} N^{-\frac{1}{2}}\right)^{\frac{1}{m+1}}$, which tends to 0 as $N \rightarrow \infty$. Therefore

$$
\liminf _{N \rightarrow \infty}\left\|\psi_{N}\right\|_{L^{\tau}(\Omega)} \geq \lim _{N \rightarrow \infty}\left\|\left(L_{m, p}\left(\frac{d^{m} f}{m !}\right)-\sqrt{\omega\left(C_{A} N^{-\frac{1}{2}}\right)}\right)_{+}\right\|_{L^{\tau}(\Omega)}=\left\|L_{m, p}\left(\frac{d^{m} f}{m !}\right)\right\|_{L^{\tau}(\Omega)} .
$$

Combining this result with 56 we conclude the proof of the announced estimate 12 . 


\subsection{A triangulation containing small periodic patches}

This subsection describes the construction of some triangulations by the aggregation of small periodic patches, which is a preliminary step for the proof of the estimate 130 of Theorem 2 in the next subsection. The design of these triangulations is related to the anterior works [4, 5, yet it is adapted in order to keep under control the measure of sliverness of the elements. The triangulations considered in this subsection are denoted by the letter $\mathcal{P}$, instead of $\mathcal{T}$, in order to avoid conflicts of notations with the next subsection.

Our first lemma describes a family of meshes of a triangle $R$, which consist for the largest part of a periodic tiling based on a triangle $T$ scaled down by a factor $1 / n$, except for a few elements close to $\partial R$.

Lemma 3.2. Let $R$ and $T$ be two triangles. There exists a family $\left(\mathcal{P}_{T, R, n}\right)_{n \geq 1}$, of conforming triangulations of $R$, and a constant $C_{R, T}$, such that the following holds.

1. (Tights bounds on the cardinality of $\mathcal{P}_{T, R, n}$ and the diameter of its elements)

$$
\lim _{n \rightarrow \infty} \frac{\#\left(\mathcal{P}_{T, R, n}\right)}{n^{2}}=\frac{|R|}{|T|} \quad \text { and } \quad \max _{T^{\prime} \in \mathcal{P}_{T, R, n}} \operatorname{diam}\left(T^{\prime}\right) \leq \frac{3}{n}(\operatorname{diam}(T)+\operatorname{diam}(R)) .
$$

2. (Conformity) The vertices of $\mathcal{P}_{T, R, n}$ on the boundary of $R$ are exactly those of the form $\frac{k}{n} a+\left(1-\frac{k}{n}\right) b$, where $0 \leq k \leq n$ and $a, b$ are vertices of $R$.

3. (Control of the boundary elements) Denote by $R_{T, n} \subset R$ the union of all the elements of $\mathcal{P}_{T, R, n}$ which are not of the following form: the image of $T$ by a map of the form $z \mapsto z_{0}+\sigma z / n$, for some $z_{0} \in \mathbb{R}^{2}, \sigma \in\{-1,1\}$. Then for all $n \geq 1$

$$
\left|R_{T, n}\right| \leq \frac{C_{R, T}}{n} \quad \text { and } \quad \max _{T^{\prime} \in \mathcal{P}_{R, T, n}} S\left(T^{\prime}\right) \leq C_{R, T}
$$

Proof. See Appendix.

We next introduce the concept of local shape specification, on the domain $\Omega$.

Definition 3.3. A local shape specification is a (possibly discontinuous) map $y \in \bar{\Omega} \mapsto T_{y}$ which associates a triangle $T_{y}$ to each point $y$ in the closure of $\Omega$, and which satisfies the following properties.

- The volume map $y \in \bar{\Omega} \mapsto\left|T_{y}\right| \in \mathbb{R}_{+}^{*}$ is continuous and positive.

- The measure of degeneracy $y \in \bar{\Omega} \mapsto \rho\left(T_{y}\right) \in \mathbb{R}_{+}$is uniformly bounded.

The next proposition describes a sequence of triangulations adapted in a certain sense to a given local shape specification. This can be compared to the construction, evoked in $\$ 1.2$, of a triangulation adapted to a given Riemmannian metric in the sense of 35 . Given a point $y \in \mathbb{R}^{2}$ and compact set $T$ we define $d_{\mathcal{H}}(y, T):=\max \{|y-z| ; z \in T\}$, which is the Haussdorf distance separating the sets $\{y\}$ and $T$.

Proposition 3.4. Let $y \mapsto T_{y}$ be a local shape specification. There exists a sequence $\left(\mathcal{P}_{n}\right)_{n \geq 2}$ of triangulations of $\Omega$, a sequence $\left(\delta_{n}\right)_{n \geq 2}$ of positive reals converging to 0 , and a constant $C_{a}$, satisfying the following properties.

1. (Tight bounds on the cardinality of $\mathcal{P}_{n}$ and the diameter of its elements)

$$
\lim _{n \rightarrow \infty} \frac{\#\left(\mathcal{P}_{n}\right)}{n^{2}}=\int_{\Omega} \frac{d y}{\left|T_{y}\right|} \quad \text { and } \quad \max _{T \in \mathcal{P}_{n}} \operatorname{diam}(T) \leq \frac{C_{a}}{n} .
$$

2. (Control of the boundary elements) Denote by $\Omega_{n} \subset \Omega$ the union of all the elements $T \in \mathcal{P}_{n}$ which are not of the following form: the image of $T_{y}$, for some $y \in \Omega$ such that $d_{\mathcal{H}}(y, T) \leq \delta_{n}$, by a map of the form $z \mapsto z_{0}+\sigma z / n$, for some $z_{0} \in \mathbb{R}^{2}, \sigma \in\{-1,1\}$. Then for all $n \geq 2$

$$
\left|\Omega_{n}\right| \leq \frac{C_{a} \ln n}{n} \quad \text { and } \quad \max _{T \in \mathcal{P}_{n}} S(T) \leq C_{a} \ln n .
$$


Proof. We consider a triangulation $\mathcal{R}^{1}$ of the polygonal domain $\Omega$ of minimal cardinality $N_{0}$. For each $k \geq 1$ we denote by $\mathcal{R}^{k}$ the triangulation of $\Omega$ of cardinality $k^{2} N_{0}$ obtained by uniformly subdividing the elements of $\mathcal{R}^{1}$ into $k^{2}$ sub triangles.

For each $n \geq 1$ we denote by $\mathcal{R}_{n}^{k}$ the triangulation of $\Omega$ obtained as the union of the triangulations $\mathcal{P}_{R, T, n}$ described in Lemma 3.2 , where $R \in \mathcal{R}^{k}$ and $T=T_{z_{R}}$ is the triangle specified by the local shape specification at the barycenter of $R$. Point 2 of Lemma 3.2 guarantees that this triangulation is conforming: there is no hanging node at the interfaces of the triangles $R \in \mathcal{R}^{k}$. Our next observation is that for each $k, n \geq 1$, using Lemma 1.1

$$
n \max _{T \in \mathcal{R}_{n}^{k}} \operatorname{diam}(T) \leq 3 \max _{R \in \mathcal{R}^{k}}\left(\operatorname{diam}(R)+\operatorname{diam}\left(T_{z_{R}}\right)\right) \leq 3 \operatorname{diam}(\Omega)+\sup _{y \in \Omega} \sqrt{\left(4 /\left|T_{\mathrm{eq}}\right|\right)\left|T_{y}\right| \rho\left(T_{y}\right)},
$$

which is finite and independent of $k$ and $n$. For each $k, n \geq 1$ we define a real $\delta(n, k)$ by the equality

$$
\frac{\#\left(\mathcal{R}_{n}^{k}\right)}{n^{2}}=\int_{\Omega} \frac{d y}{\left|T_{y}\right|}+\delta(n, k)
$$

Using (57) we obtain

$$
\lim _{n \rightarrow \infty} \delta(n, k)=\delta(k):=\sum_{R \in \mathcal{R}^{k}} \frac{|R|}{\left|T_{z_{R}}\right|}-\int_{\Omega} \frac{d y}{\left|T_{y}\right|}
$$

Note that $\delta(k) \rightarrow 0$ as $k \rightarrow \infty$ since the map $y \mapsto T_{y}$ is continuous.

We denote by $\Omega_{n}^{k}$ the union of the sets $R_{T, n}$ described in Lemma 3.2 for $R \in \mathcal{R}^{k}$ and $T=T_{z_{R}}$, and by $C_{k}$ the sum of the corresponding constants $C_{R, T}$. We obtain using (58) that for all $n \geq 1$

$$
\left|\Omega_{n}^{k}\right| \leq \frac{C_{k}}{n} \quad \text { and } \quad \max _{T \in \mathcal{R}_{n}^{k}} S(T) \leq C_{k}
$$

We finally choose a sequence $(k(n))_{n \geq 2}$, such that $k(n) \rightarrow \infty$ as $n \rightarrow \infty$, and which increases "slowly" in the following sense: we require that for $n$ sufficiently large one has $\delta(n, k(n)) \leq \delta(k(n))+\frac{1}{n}$ and $C_{k(n)} \leq \ln n$. Defining $\mathcal{P}_{n}:=\mathcal{R}_{n}^{k(n)}$ and $\delta_{n}:=\operatorname{diam}(\Omega) / k(n)$ we obtain the announced result.

\subsection{The upper error estimate (13)}

Throughout this section we consider a fixed real $M \geq 1$, and we introduce the collection of triangles

$$
\mathbf{T}_{M}:=\left\{T ;|T|=1, \rho(T) \leq M, z_{T}=0\right\}
$$

which is compact for the Haussdorf distance. We introduce a variant $L_{M}$ of the shape function $L_{m, p}$ defined as follows: for each $\pi \in \mathbb{H}_{m}$

$$
L_{M}(\pi):=\min _{T \in \mathbf{T}_{M}}\left\|\nabla\left(\pi-\mathrm{I}_{T}^{m-1} \pi\right)\right\|_{L_{p}(T)} .
$$

For any fixed $\pi \in \mathbb{H}_{m}$ the map $T \mapsto\left\|\nabla\left(\pi-\mathrm{I}_{T}^{m-1} \pi\right)\right\|_{L^{p}(T)}$ is continuous with respect to the Haussdorff distance on the set of all triangles. Hence there exists a minimizing triangle, that we denote by $T(\pi) \in$ $\mathbf{T}_{M}$, for the optimization problem appearing in 60 .

Since all norms are equivalent on the finite dimensional space $\mathbb{H}_{m}$, there exists a constant $C_{M}$ such that for all $\pi \in \mathbb{H}_{m}$

$$
\sup _{T \in \mathbf{T}_{M}}\left\|\nabla\left(\pi-\mathrm{I}_{T}^{m-1} \pi\right)\right\|_{L^{p}(T)} \leq C_{M}\|\nabla \pi\| .
$$

The function $L_{M}$ is defined as the infimum of a family of $C_{M}$-Lipschitz functions on $\mathbb{H}_{m}$, hence is also $C_{M}$-Lipschitz: $\left|L_{M}(\pi)-L_{M}\left(\pi^{\prime}\right)\right| \leq C_{M}\left\|\nabla \pi-\nabla \pi^{\prime}\right\|$. Furthermore for each $\pi, \pi^{\prime} \in \mathbb{H}_{m}$, one has since $T(\pi) \in \mathbf{T}_{M}$

$$
\left\|\nabla\left(\pi^{\prime}-\mathrm{I}_{T(\pi)}^{m-1} \pi^{\prime}\right)\right\|_{L^{p}(T(\pi))} \leq\left\|\nabla\left(\pi-\mathrm{I}_{T(\pi)}^{m-1} \pi\right)\right\|_{L^{p}(T(\pi))}+C_{M}\left\|\pi-\pi^{\prime}\right\|=L_{M}(\pi)+C_{M}\left\|\pi-\pi^{\prime}\right\| .
$$


We consider the following local shape specification, see Definition 3.3 .

$$
y \mapsto T_{y}:=\left(L_{M}\left(\pi_{y}\right)+M^{-1}\right)^{-\frac{\tau}{2}} T\left(\pi_{y}\right) .
$$

In other words $T_{y}$ is the isotropic scaling of the triangle $T\left(\pi_{y}\right)$ by the factor $\left(L_{M}\left(\pi_{y}\right)+M^{-1}\right)^{-\frac{\tau}{2}}$. We thus have $\left|T_{y}\right|=\left(L_{M}\left(\pi_{y}\right)+M^{-1}\right)^{-\tau}$, which depends continuously on $y \in \bar{\Omega}$, and $\rho\left(T_{y}\right)=\rho\left(T\left(\pi_{y}\right)\right) \leq M$.

Proposition 3.4 describes a sequence $\left(\mathcal{P}_{n}\right)_{n \geq 2}$ of triangulations of $\Omega$ attached to this local shape specification, as well as a sequence $\left(\delta_{n}\right)_{n \geq 2}$ of positive numbers, a sequence $\left(\Omega_{n}\right)_{n \geq 2}$ of subdomains of $\Omega$, and a constant $C_{a}$. We recall that for all $n \geq 2$ and all $T \in \mathcal{P}_{n}$

$$
\operatorname{diam}(T) \leq C_{a} / n, \quad S(T) \leq C_{a} \ln n, \quad\left|\Omega_{n}\right| \leq C_{a}(\ln n) / n .
$$

Using Point ii of Lemma 3.1 we obtain for each $n \geq 1$ and each triangle $T \in \mathcal{P}_{n}$

$$
\left\|\nabla\left(f-\mathrm{I}_{T}^{m-1} f\right)\right\|_{L^{p}(T)} \leq C_{\star} C_{a}^{m}|T|^{\frac{1}{p}}(\ln n) / n^{m-1} .
$$

Summing up the $p$-th power of the contributions of all the triangles $T \in \mathcal{P}_{n}$ such that $T \subset \Omega_{n}$, we obtain

$$
\left\|\nabla\left(f-\mathrm{I}_{T}^{m-1} f\right)\right\|_{L^{p}\left(\Omega_{n}\right)} \leq C_{\star} C_{a}^{m}\left|\Omega_{n}\right|^{\frac{1}{p}} \frac{\ln n}{n^{m-1}} \leq \frac{C_{\star} C_{a}^{m+1}(\ln n)^{1+\frac{1}{p}} n^{-\frac{1}{p}}}{n^{m-1}}=: \frac{\varepsilon_{n}}{n^{m-1}},
$$

and we observe that $\varepsilon_{n} \rightarrow 0$ as $n \rightarrow \infty$.

We now turn to the contribution of $\Omega \backslash \Omega_{n}$ to the error, and for that purpose we consider a triangle $T$ which is the image of the triangle $T_{y}$, for some $y \in \Omega$ such that $d_{\mathcal{H}}(y, T) \leq \delta_{n}$, by a map of the form $z \mapsto z_{0}+\sigma z / n$, for some $z_{0} \in \mathbb{R}^{2}, \sigma \in\{-1,1\}$. We have for any $z \in T$, using Point i of Lemma 3.1

$$
\begin{aligned}
\left\|\nabla\left(f-\mathrm{I}_{T}^{m-1} f\right)\right\|_{L^{p}(T)} & \leq\left\|\nabla\left(\pi_{z}-\mathrm{I}_{T}^{m-1} \pi_{z}\right)\right\|_{L^{p}(T)}+C_{\star} \omega\left(C_{a} / n\right)|T|^{\frac{1}{\tau}} M^{\frac{m+1}{2}} \\
& =|T|^{\frac{1}{\tau}}\left(\left\|\nabla\left(\pi_{z}-\mathrm{I}_{T\left(\pi_{y}\right)}^{m-1} \pi_{z}\right)\right\|_{L^{p}\left(T\left(\pi_{y}\right)\right)}+C_{\star} \omega\left(C_{a} / n\right) M^{\frac{m+1}{2}}\right) \\
& \leq \frac{L_{M}\left(\pi_{y}\right)+C_{M} \omega\left(\delta_{n}\right)+C_{\star} M^{\frac{m+1}{2}} \omega\left(C_{a} / n\right)}{n^{\frac{2}{\tau}}\left(L_{M}\left(\pi_{y}\right)+M^{-1}\right)},
\end{aligned}
$$

where we used the invariance property $(8)$ in the second line, and $\sqrt{61}$ in the last line. It follows that there exists an integer $n_{0}$ such that $\left\|\nabla\left(f-\mathrm{I}_{T}^{m-1} f\right)\right\|_{L^{p}(T)} \leq n^{-\frac{2}{\tau}}$ for all $n \geq n_{0}$ and all $T \in \mathcal{P}_{n}$. Hence

$$
\begin{aligned}
\left\|\nabla\left(f-\mathrm{I}_{\mathcal{P}_{n}}^{m-1} f\right)\right\|_{L^{p}(\Omega)} & \leq\left\|\nabla\left(f-\mathrm{I}_{\mathcal{P}_{n}}^{m-1} f\right)\right\|_{L^{p}\left(\Omega \backslash \Omega_{n}\right)}+\left\|\nabla\left(f-\mathrm{I}_{\mathcal{P}_{n}}^{m-1} f\right)\right\|_{L^{p}\left(\Omega_{n}\right)} \\
& \leq \#\left(\mathcal{P}_{n}\right)^{\frac{1}{p}} n^{-\frac{2}{\tau}}+\varepsilon_{n} n^{-(m-1)}
\end{aligned}
$$

and therefore using $[59]$

$$
\begin{aligned}
\limsup _{n \rightarrow \infty} \#\left(\mathcal{P}_{n}\right)^{\frac{m-1}{2}}\left\|\nabla\left(f-\mathrm{I}_{\mathcal{P}_{n}}^{m-1} f\right)\right\|_{L^{p}(\Omega)} & \leq \lim _{n \rightarrow \infty} \frac{\#\left(\mathcal{P}_{n}\right)^{\frac{1}{\tau}}}{n^{\frac{2}{\tau}}}+\frac{\#\left(\mathcal{P}_{n}\right)^{\frac{m-1}{2}} \varepsilon_{n}}{n^{m-1}} \\
& =\left(\int_{\Omega} \frac{d y}{\left|T_{y}\right|}\right)^{\frac{1}{\tau}}+0 \\
& =\left(\int_{\Omega}\left(L_{M}\left(\pi_{y}\right)+M^{-1}\right)^{\tau} d y\right)^{\frac{1}{\tau}} .
\end{aligned}
$$

Observe that $L_{M}\left(\pi_{y}\right)$ converges decreasingly for each $y \in \Omega$ to $L_{m, p}\left(d^{m} f(y) / m !\right):=L_{m, p}\left(\pi_{y}\right)$ as $M \rightarrow \infty$. Given any fixed $\varepsilon>0$, and using standard results on the convergence of integrals, we may therefore choose $M=M(\varepsilon)$ sufficiently large such that

$$
\left(\int_{\Omega}\left(L_{M}\left(\pi_{y}\right)+M^{-1}\right)^{\tau} d y\right)^{\frac{1}{\tau}} \leq\left\|L_{m, p}\left(\frac{d^{m} f}{m !}\right)\right\|_{L^{\tau}(\Omega)}+\varepsilon .
$$

We denote by $N_{0}$ the minimal cardinality of a triangulation of the polygonal domain $\Omega$, and we assume without loss of generality that $\#\left(\mathcal{P}_{2}\right)=N_{0}$. For each $N \geq N_{0}$, we denote by $n(N)$ the largest integer 
such that $\#\left(\mathcal{P}_{n(N)}\right) \leq N$, and we set $\mathcal{T}_{N}^{\varepsilon}:=\mathcal{P}_{n(N)}$. Observing that $\#\left(\mathcal{T}_{N}^{\varepsilon}\right) / N \rightarrow 1$ as $N \rightarrow \infty$, and combining 62 with 63 , we obtain the announced result 13 :

$$
\limsup _{N \rightarrow \infty} N^{\frac{m-1}{2}}\left\|\nabla\left(f-\mathrm{I}_{\mathcal{T}_{N}^{\varepsilon}}^{m-1} f\right)\right\|_{L^{p}(\Omega)}=\limsup _{n \rightarrow \infty} \#\left(\mathcal{P}_{n(N)}\right)^{\frac{m-1}{2}}\left\|\nabla\left(f-\mathrm{I}_{\mathcal{P}_{n(N)}-1}^{m-1} f\right)\right\|_{L^{p}(\Omega)} \leq\left\|L_{m, p}\left(\frac{d^{m} f}{m !}\right)\right\| \|_{L^{\tau}(\Omega)}+\varepsilon
$$

The admissibility of the sequence $\left(\mathcal{T}_{N}^{\varepsilon}\right)_{N \geq N_{0}}$ of triangulations immediately follows from $(59)$.

\section{Conclusion}

In this paper, we have introduced asymptotic estimates for the finite element interpolation error measured in the $W^{1, p}$ semi-norm, when the mesh is optimally adapted to a function of two variables and the degree of interpolation $m-1$ is arbitrary. The approach used is an adaptation of the ideas developped in [18] for the $L^{p}$ interpolation error, and leads to asymptotically sharp error estimates, exposed in Theorems 1 and 2. These estimates involve a shape function $L_{m, p}$ which generalises the determinant which appears in estimates for piecewise linear interpolation. The shape function has equivalents of polynomial form for all values of $m$, as established in $\$ 2.2$. Up to a fixed multiplicative constant, our estimates can therefore be written under analytic form in terms of the derivatives of the function to be approximated.

Future efforts will be devoted to the extension of these results to functions defined on a domain of dimension $d>2$, which is partially done in Chapter 3 of 20]. Another challenge left open is the development of an anisotropic mesh generator with guarantees on the maximal angle of the elements, as evoked in $\$ 1.2$, which could allow to apply the results of this paper in the context of adaptive mesh refinement for numerical simulations.

\section{APPENDIX}

\section{A Proof of Lemma 3.2}

We construct the triangulation $\mathcal{P}_{T, R, n}$ of $R$ as the union of three components: $\mathcal{P}_{T, R, n}=\mathcal{B}_{n} \cup \mathcal{L}_{n} \cup \mathcal{I}_{n}$. The elements of $\mathcal{B}_{n}$ cover the boundary of $R$, while the elements of $\mathcal{I}_{n}$ cover most of its interior and are included in $\overline{R \backslash R_{T, n}}$. The elements of $\mathcal{L}_{n}$ play the role of a layer between $\mathcal{I}_{n}$ and $\mathcal{B}_{n}$. Throughout this proof we denote by $C=C(R, T)$ a generic constant independent of $n$, which may change from one occurrence to the next.

We introduce the homothetic contraction $R_{n}$ of the triangle $R$ by the factor $1-n^{-1}$, with the same barycenter (i.e. the image of $R$ by the map $\left.z \mapsto z_{R}+\left(1-n^{-1}\right)\left(z-z_{R}\right)\right)$. One easily checks that for all $z \in \partial R$

$$
\frac{2}{3} \frac{|R|}{\operatorname{diam}(R)} \leq n d\left(z, R_{n}\right) \leq \frac{2}{3} \operatorname{diam}(R)
$$

where $d(z, E):=\inf \{|z-e| ; e \in E\}$ for any $z \in \mathbb{R}^{2}$ and any $E \subset \mathbb{R}^{2}$.

Let $z_{0}$ be a vertex of the triangle $T$, and let $u, v$ be two of its edge vectors. For each $n \geq 1$ we denote by $\mathcal{I}_{n}^{0}$ the periodic tiling of $\mathbb{R}^{2}$ built of the images of $T$ by the maps $z \mapsto \alpha u+\beta v+\left(z_{0}+\sigma\left(z-z_{0}\right)\right) / n$, where $\alpha, \beta \in \mathbb{Z}$ and $\sigma \in\{-1,1\}$. This tiling is built of translations of the triangle $T$, and of its symmetric with respect to the vertex $z_{0}$, scaled by the factor $1 / n$. We define the collection of triangles

$$
\mathcal{I}_{n}:=\left\{T^{\prime} \in \mathcal{I}_{n}^{0} ; T^{\prime} \subset R_{n}\right\}
$$

which is illustrated in dark gray on Figure 3. Any $T^{\prime} \in \mathcal{I}_{n}$ satisfies $\operatorname{diam}\left(T^{\prime}\right)=\operatorname{diam}(T) / n$ and $S\left(T^{\prime}\right)=$ $S(T)$. Furthermore these elements are of the form mentioned in Point 3 of the lemma, hence are included in $\overline{R \backslash R_{T, n}}$. The elements of $\mathcal{I}_{n}$ cover the set $\{y \in R ; n d(y, \partial R) \geq \operatorname{diam}(T)+\operatorname{diam}(R)\}$, which area is larger than $|R|-C / n$. We thus obtain the left part of (58). Observing that $\left|T^{\prime}\right|=|T| / n^{2}$ for each $T^{\prime} \in \mathcal{T}^{\prime}$, we obtain that $\#\left(\mathcal{I}_{n}\right) / n^{2} \rightarrow|R| /|T|$ as $n \rightarrow \infty$.

We define a collection $\mathcal{L}_{n}^{0}$ of convex polygons as follows:

$$
\mathcal{L}_{n}^{0}:=\left\{R_{n} \cap T^{\prime} ; T^{\prime} \in \mathcal{I}_{n}^{0} \text { and } \operatorname{int}\left(T^{\prime}\right) \cap \partial R_{n} \neq \emptyset\right\},
$$



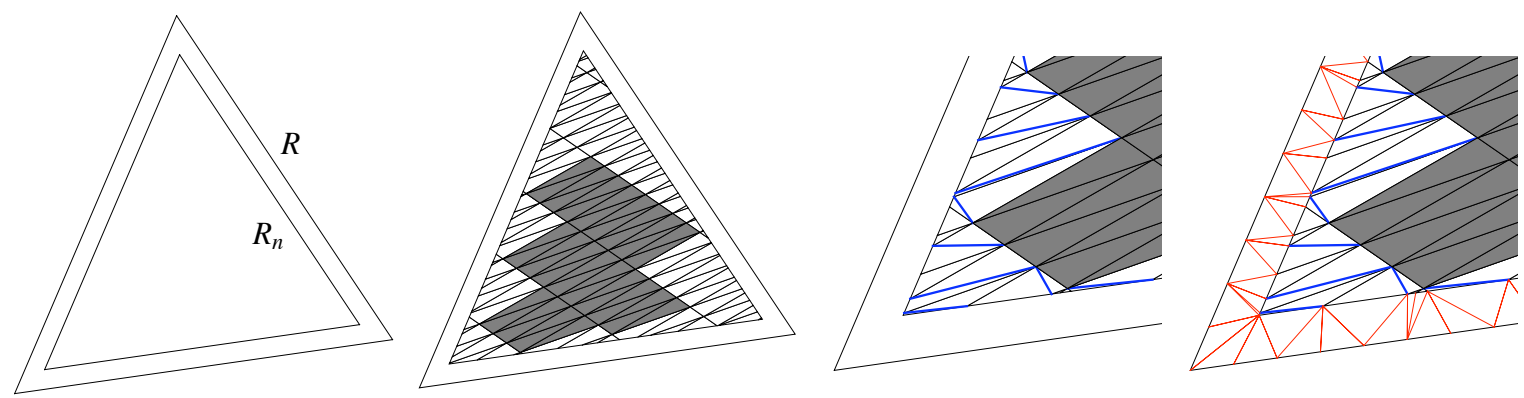

Figure 3: The triangles $R$ and $R_{n}$ (left). The collections $\mathcal{I}_{n}$ (gray) of triangles and $\mathcal{L}_{n}^{0}$ (white) of convex polygons (center left). The collection $\mathcal{L}_{n}$ of triangles is obtained by triangulating the elements of $\mathcal{L}_{n}^{0}$ (center right). The collection $\mathcal{B}_{n}$ of triangles covers $R \backslash R_{n}$ (right).

where $\operatorname{int}(E)$ denotes the interior of a set $E \subset \mathbb{R}^{2}$. The set $\mathcal{L}_{n}^{0}$ is illustrated in white on Figure 3 (center left). The elements $T^{\prime} \in \mathcal{I}_{n}^{0}$ such that $\operatorname{int}\left(T^{\prime}\right) \cap \partial R_{n} \neq \emptyset$ are included in the set $\left\{y \in \mathbb{R}^{2} ; n d(y, \partial R) \leq\right.$ $\operatorname{diam}(R)+\operatorname{diam}(T)\}$ which area is smaller than $C / n$, and their individual area is $|T| / n^{2}$. Therefore $\#\left(\mathcal{L}_{n}^{0}\right) \leq C n$. The normals to faces of the elements of $\mathcal{L}_{n}^{0}$ belong to a family of at most 6 elements: the normals to the faces of $T$, and to the faces of $R$. Therefore at most $6 \times 5$ different angles can appear in $\mathcal{L}_{n}^{0}$, and we denote the largest of these by $\alpha<\boldsymbol{\pi}$. We denote by $\mathcal{L}_{n}$ the collection of triangles, illustrated Figure 3 (center right), obtained by triangulating each element of $\mathcal{L}_{n}^{0}$, which is a convex polygon with at most six faces. The angles of the triangles partitioning a convex polygon are smaller than the angles of this polygon, hence the angles of the elements of $\mathcal{L}_{n}$ are also bounded by $\alpha$. Furthermore $\#\left(\mathcal{L}_{n}\right) \leq 4 \#\left(\mathcal{L}_{n}^{0}\right) \leq C n$, and $\operatorname{diam}\left(T^{\prime}\right) \leq \operatorname{diam}(T) / n$ for each $T^{\prime} \in \mathcal{L}_{n}$.

We denote by $E_{n}$ the collection of $n$ equidistributed points on each edge of $R$, described in Point 2 of Lemma 3.2 and we denote by $E_{n}^{\prime}$ the collection of vertices of the triangles in $\mathcal{L}_{n}$ that fall on $\partial R_{n}^{\prime}$. For each point $p \in E_{n}$, we draw an edge between $p$ and the point of $p^{\prime} \in E_{n}^{\prime}$ which is the closest to $p$. Note that $\left|p-p^{\prime}\right| \leq d\left(p, R_{n}\right)+\operatorname{diam}(T) / n \leq(\operatorname{diam}(R)+\operatorname{diam}(T)) / n$. This produces a partition $\mathcal{B}_{n}^{0}$ of $\overline{R \backslash R_{n}}$ into triangles and convex quadrilaterals, of diameter at most

$$
\operatorname{diam}(R) / n+2(\operatorname{diam}(R)+\operatorname{diam}(T)) / n \leq 3(\operatorname{diam}(R)+\operatorname{diam}(T)) / n,
$$

since the distance between two consecutive points in $E_{n}$ is at most $\operatorname{diam}(R)$. We denote by $\mathcal{B}_{n}$ the collection of triangles, illustrated Figure 3 (right), obtained by triangulating each polygon $K \in \mathcal{B}_{n}^{0}$, of vertices $\bar{K} \cap\left(E_{n} \cup E_{n}^{\prime}\right)$. We have $\#\left(\mathcal{B}_{n}\right)=\#\left(E_{n}\right)+\#\left(E_{n}^{\prime}\right) \leq 3 n+3 \#\left(\mathcal{L}_{n}^{0}\right) \leq C n$. In order to conclude the proof of this lemma, we only need to show that the angles of the elements of $\mathcal{B}_{n}$ are uniformly bounded away from $\boldsymbol{\pi}$.

We consider a triangle $T^{\prime} \in \mathcal{B}_{n}$, we denote by $L$ the length of the edge of $T^{\prime}$ included in $\partial R \cup \partial R_{n}$, and by $H$ the height of the triangle $T^{\prime}$ such that $L H=2\left|T^{\prime}\right|$. It follows from (64) that $H \geq 2|T| /(3 n \operatorname{diam}(T))$. Let $L^{\prime}$ be another edge of $T^{\prime}$, and let $\theta$ be the angle of $T^{\prime}$ between the edges $L$ and $L^{\prime}$. Then

$$
2\left|T^{\prime}\right|=L L^{\prime} \sin \theta=L H,
$$

hence $\sin \theta \geq \frac{H}{\operatorname{diam}\left(T^{\prime}\right)} \geq \frac{c}{C}$, where $c=2|T| /(3 n \operatorname{diam}(T))$ and $C=3(\operatorname{diam}(R)+\operatorname{diam}(T))$, which implies that $\arcsin \left(\frac{c}{C}\right) \leq \theta \leq \pi-\arcsin \left(\frac{c}{C}\right)$. It follows that all the angles of $T^{\prime}$ are smaller than $\pi-\arcsin \left(\frac{c}{C}\right)$ which concludes the proof.

\section{B Proof of Theorem 2.5}

We consider an arbitrary but fixed $r \geq 2$ and we define $s_{r}:=\left\lfloor\frac{r}{2}\right\rfloor+1$. It is established in Proposition 2.1 of [18] (equivalently Proposition 2.2.1 of [20]) that for any $\pi \in \mathbb{H}_{r}$ the three following properties are equivalent

$\left[K_{r}(\pi)=0\right.$,

There exists $\alpha, \beta \in \mathbb{R}$ and $\tilde{\pi} \in \mathbb{H}_{r-s_{r}}$ such that $\pi=(\alpha x+\beta y)^{s_{r}} \tilde{\pi}$,

There exists a sequence $\left(\phi_{n}\right)_{n \geq 0}, \phi_{n} \in \mathrm{SL}_{2}$, such that $\pi \circ \phi_{n} \rightarrow 0$. 
In addition the following invariance property is established in Theorem 2.6.3 of [20]: let $Q$ be a polynomial on $\mathbb{H}_{r}$ such that $K_{r} \sim \sqrt[d]{|Q|}$ where $d=\operatorname{deg} Q$. Then $d r / 2$ is an integer and for all $\pi \in \mathbb{H}_{r}$ and all $\phi \in \mathrm{M}_{2}$

$$
Q(\pi \circ \phi)=(\operatorname{det} \phi)^{\frac{d r}{2}} Q(\pi) .
$$

It follows that the polynomials $\left(Q_{k}\right)_{0 \leq k \leq d}$, defined in (47), satisfy for all $\pi_{1}, \pi_{2} \in \mathbb{H}_{r}$ and all $\phi \in \mathrm{M}_{2}$

$$
Q_{k}\left(\pi_{1} \circ \phi, \pi_{2} \circ \phi\right)=(\operatorname{det} \phi)^{\frac{d r}{2}} Q_{k}\left(\pi_{1}, \pi_{2}\right) .
$$

We define two functions on $\mathbb{H}_{r} \times \mathbb{H}_{r}$

$$
K_{*}\left(\pi_{1}, \pi_{2}\right):=\sqrt[2 d]{\sum_{0 \leq k \leq r} Q_{k}\left(\pi_{1}, \pi_{2}\right)^{2}} \text { and } K\left(\pi_{1}, \pi_{2}\right):=\sqrt[2 \tilde{d}]{\tilde{Q}\left(\pi_{1}^{2}+\pi_{2}^{2}\right)}
$$

where $\tilde{Q}$ is an homogeneous polynomial on $\mathbb{H}_{2 r}$ such that $K_{2 r} \sim \sqrt[\tilde{d}]{\tilde{Q}}$, and $\tilde{d}:=\operatorname{deg} \tilde{Q}$. We show below that $K \sim K_{*}$ on $\mathbb{H}_{r} \times \mathbb{H}_{r}$. Choosing $r=m-1$ and combining this result with (44) concludes the proof of Theorem 2.5 .

Using $(67)$ and remarking the invariance property $\tilde{Q}(\pi \circ \phi)=(\operatorname{det} \phi)^{\tilde{d} r} Q(\pi)$, for the same reasons as (66), we obtain for all $\pi_{1}, \pi_{2} \in \mathbb{H}_{r}$ and all $\phi \in \mathrm{M}_{2}$

$$
\begin{aligned}
K\left(\pi_{1} \circ \phi, \pi_{2} \circ \phi\right) & =|\operatorname{det} \phi|^{\frac{r}{2}} K\left(\pi_{1}, \pi_{2}\right), \\
K_{*}\left(\pi_{1} \circ \phi, \pi_{2} \circ \phi\right) & =|\operatorname{det} \phi|^{\frac{r}{2}} K_{*}\left(\pi_{1}, \pi_{2}\right) .
\end{aligned}
$$

Consider $\pi_{1}, \pi_{2} \in \mathbb{H}_{r}$. The equality $K\left(\pi_{1}, \pi_{2}\right)=0$ is equivalent to $\pi_{1}^{2}+\pi_{2}^{2} \in \mathbb{H}_{2 r}$ having a linear factor of multiplicity $s_{2 r}=r+1$ (according to (65)), which is also equivalent to $\pi_{1}$ and $\pi_{2}$ having a common linear factor of multiplicity $s_{r}$.

On the other hand the equality $K_{*}\left(\pi_{1}, \pi_{2}\right)=0$ is equivalent to $Q_{k}\left(\pi_{1}, \pi_{2}\right)=0$ for all $0 \leq k \leq d$. This is equivalent to $K_{r}\left(u \pi_{1}+v \pi_{2}\right)=0$ for all $u, v \in \mathbb{R}$ (using (47)), which means that the polynomial $u \pi_{1}+v \pi_{2} \in \mathbb{H}_{r}$ has a linear factor of multiplicity $s_{r}$ for all $u, v \in \mathbb{R}$ (using (65)). This is equivalent to $\pi_{1}$ and $\pi_{2}$ having a common linear factor of multiplicity $s_{r}$.

The following properties are therefore equivalent

$$
\left[\begin{array}{l}
K\left(\pi_{1}, \pi_{2}\right)=0, \\
K_{*}\left(\pi_{1}, \pi_{2}\right)=0, \\
\text { There exists } \alpha, \beta \in \mathbb{R} \text { and } \tilde{\pi}_{1}, \tilde{\pi}_{2} \in \mathbb{H}_{r-s_{r}} \text { such that } \pi_{1}=(\alpha x+\beta y)^{s_{r}} \tilde{\pi}_{1} \text {, and } \pi_{2}=(\alpha x+\beta y)^{s_{r}} \tilde{\pi}_{2} .
\end{array}\right.
$$

Using $(65)$ and the definition 68 of $K$, we find that these properties are also equivalent to

$$
\left[\begin{array}{l}
K_{2 r}\left(\pi_{1}^{2}+\pi_{2}^{2}\right)=0, \\
\text { There exists a sequence }\left(\phi_{n}\right)_{n \geq 0}, \phi_{n} \in \mathrm{SL}_{2}, \text { such that }\left(\pi_{1} \circ \phi_{n}\right)^{2}+\left(\pi_{2} \circ \phi_{n}\right)^{2} \rightarrow 0, \\
\text { There exists a sequence }\left(\phi_{n}\right)_{n \geq 0}, \phi_{n} \in \mathrm{SL}_{2}, \text { such that } \pi_{1} \circ \phi_{n} \rightarrow 0 \text { and } \pi_{2} \circ \phi_{n} \rightarrow 0 .
\end{array}\right.
$$

We now define the norm $\left\|\left(\pi_{1}, \pi_{2}\right)\right\|:=\sup _{|u| \leq 1}\left|\left(\pi_{1}(u), \pi_{2}(u)\right)\right|$ on $\mathbb{H}_{r} \times \mathbb{H}_{r}$, and the set

$$
\mathcal{F}:=\left\{\left(\pi_{1}, \pi_{2}\right) \in \mathbb{H}_{r} \times \mathbb{H}_{r} ;\left\|\left(\pi_{1}, \pi_{2}\right)\right\|=1 \text { and }\left\|\left(\pi_{1} \circ \phi, \pi_{2} \circ \phi\right)\right\| \geq 1 \text { for all } \phi \in \mathrm{SL}_{2}\right\} .
$$

The set $\mathcal{F}$ is compact subset of $\mathbb{H}_{r} \times \mathbb{H}_{r}$, and $K$ as well as $K_{*}$ do not vanish on $\mathcal{F}$ according to 70 and 711. Since these functions are continuous, there exists a constant $C_{0} \geq 1$ such that

$$
C_{0}^{-1} K \leq K_{*} \leq C_{0} K \text { on } \mathcal{F} \text {. }
$$

Let $\left(\pi_{1}, \pi_{2}\right) \in \mathbb{H}_{r} \times \mathbb{H}_{r}$. If there exists a sequence $\left(\phi_{n}\right)_{n>0}, \phi_{n} \in \mathrm{SL}_{2}$, such that $\pi_{1} \circ \phi_{n} \rightarrow 0$ and $\pi_{2} \circ \phi_{n} \rightarrow 0$, then $K\left(\pi_{1}, \pi_{2}\right)=K(0,0)=0$ and $K_{*}\left(\pi_{1}, \pi_{2}\right)=K_{*}(0,0)=0$ using (69) and the continuity of $K$ and $K_{*}$. Otherwise, consider a sequence $\left(\phi_{n}\right)_{n \geq 0}, \phi_{n} \in \mathrm{SL}_{2}$, such that

$$
\lim _{n \rightarrow \infty}\left\|\left(\pi_{1} \circ \phi_{n}, \pi_{2} \circ \phi_{n}\right)\right\|=\inf _{\phi \in \mathrm{SL}_{2}}\left\|\left(\pi_{1} \circ \phi, \pi_{2} \circ \phi\right)\right\| .
$$


By compactness there exists a pair $\left(\tilde{\pi}_{1}, \tilde{\pi}_{2}\right) \in \mathbb{H}_{m} \times \mathbb{H}_{m}$ and a subsequence $\left(\phi_{n_{k}}\right)_{k \geq 0}$ such that

$$
\left(\pi_{1} \circ \phi_{n_{k}}, \pi_{2} \circ \phi_{n_{k}}\right) \rightarrow\left(\tilde{\pi}_{1}, \tilde{\pi}_{2}\right) .
$$

One easily checks that $\frac{\left(\tilde{\pi}_{2}, \tilde{\pi}_{2}\right)}{\left\|\left(\tilde{\pi}_{2}, \tilde{\pi}_{2}\right)\right\|} \in \mathcal{F}$. Using 69 we obtain

$$
\frac{K\left(\pi_{1}, \pi_{2}\right)}{K_{*}\left(\pi_{1}, \pi_{2}\right)}=\lim _{n \rightarrow \infty} \frac{K\left(\pi_{1} \circ \phi_{n}, \pi_{2} \circ \phi_{n}\right)}{K_{*}\left(\pi_{1} \circ \phi_{n}, \pi_{2} \circ \phi_{n}\right)}=\frac{K\left(\tilde{\pi}_{1}, \tilde{\pi}_{2}\right)}{K_{*}\left(\tilde{\pi}_{1}, \tilde{\pi}_{2}\right)} .
$$

Using (72) and the homogeneity of $K$ and $K_{*}$, we obtain that $C_{0}^{-1} K \leq K_{*} \leq C_{0} K$ on $\mathbb{H}_{r} \times \mathbb{H}_{r}$ which concludes the proof.

\section{Acknowledgement}

I am extremely grateful to my Ph.D advisor Albert Cohen for his support in the elaboration of this paper.

\section{References}

[1] G. Acosta, T. Apel, Ricardo G. Durân, Ariel L. Lombardi, Anisotropic error estimates for an interpolant defined via moments, Computing, 82(2008), 1-9.

[2] T. Apel, M. Berzins, P.K. Jimack, G. Kunert, A. Plaks, I. Tsukerman, M. Walkley, Mesh shape and anisotropic elements: theory and practice, The mathematics of finite elements and applications, $\mathrm{X}$, MAFELAP 1999 (Uxbridge), 367-376, Elsevier, Oxford, 2000.

[3] E. F. D'Azevedo and R. B. Simpson, On optimal regular meshes for minimizing the gradient error, Numer. Math., 59:321-348, 1991.

[4] V. Babenko, Y. Babenko, A. Ligun and A. Shumeiko, On Asymptotical Behavior of the Optimal Linear Spline Interpolation Error of $C^{2}$ Functions, East J. Approx. 12(1), 71-101, 2006.

[5] Y. Babenko, T. Leskevich, J.-M. Mirebeau, Sharp asymptotics of the $L_{p}$ approximation error for interpolation on block partitions, Numerische Mathematik, 2010.

[6] I. Babuška, A. K. Aziz On the angle condition in the finite element method, SIAM J. Numer. Anal. 13, 1976

[7] J-D. Boissonnat, C. Wormser and M. Yvinec. Locally uniform anisotropic meshing, Proceedings of the twenty-fourth annual symposium on Computational geometry, june 2008 (SOCG 2008)

[8] W. Cao. An interpolation error estimate on anisotropic meshes in $\mathbb{R}^{n}$ and optimal metrics for mesh refinement. SIAM J. Numer. Anal. 45 no. 6, 2368-2391, 2007.

[9] W. Cao. On the error of linear interpolation and the orientation, aspect ratio, and internal angles of a triangle, SIAM J. Numer. Anal., 43(1), 19-40, 2005.

[10] W. Cao. Anisotropic measures of third order derivatives and the quadratic interpolation error on triangular elements, SIAM J. Sci. Comput., 29(2), 756-781 (electronic), 2007.

[11] L. Chen, P. Sun and J. Xu, Optimal anisotropic meshes for minimizing interpolation error in $L^{p}$ norm, Math. of Comp. 76, 179-204, 2007.

[12] A. Cohen, J.-M. Mirebeau, Adaptive and anisotropic piecewise polynomial approximation, chapter 4 of the book Multiscale, Nonlinear and Adaptive Approximation, Springer, 2009

[13] L. Formaggia, S. Perotto, New anisotropic a priori error estimates, Numerische Mathematik 89, pp 641-667, 2001 
[14] W. Huang and W. Sun, Variational mesh adaptation II: Error estimates and monitor functions, Journal of Computational Physics, 184:619-648, 2003.

[15] P. Jamet Estimations d'erreur pour des éléments finis droits presque dégénérés, CRM-447, Centre d'Etudes de Limiel.

[16] F. Labelle and J. R. Shewchuk, Anisotropic Voronoi Diagrams and Guaranteed-Quality Anisotropic Mesh Generation, Proceedings of the Nineteenth AnnualSymposium on Computational Geometry, 191-200, 2003.

[17] A. Loseille and F. Alauzet, Continuous mesh framework part I: well-posed continuous interpolation error, SIAM J. Numer. Anal. 49 (2011), no. 1, 38-60.

[18] J.-M. Mirebeau, Optimal meshes for finite elements of arbitrary order, Constructive Approximation, Vol $32 \mathrm{n}^{\circ} 2$, pages 339-383, 2010.

[19] J.-M. Mirebeau, The optimal aspect ratio for piecewise quadratic anisotropic finite element approximation, proceedings of the conference SampTA 2011 (submitted)

[20] J.-M. Mirebeau, Adaptive and anisotropic finite element approximation: Theory and algorithms, $\mathrm{PhD}$ Thesis, tel.archives-ouvertes.fr/tel-00544243/en/

[21] J. R. ShewChuk, What is a good linear finite element? Interpolation, Conditioning, Anisotropy, and Quality Measures, Proceedings of the 11th International Meshing Roundtable, 2002

[22] FreeFem++ software, developped by Frederic Hecht, www.freefem.org/ff++/

[23] A 3-d anisotropic mesh generator: www.math.u-bordeaux1.fr/ \{\}dobj/logiciels/mmg3d.php

Jean-Marie Mirebeau

UPMC Univ Paris 06, UMR 7598, Laboratoire Jacques-Louis Lions, F-75005, Paris, France

CNRS, UMR 7598, Laboratoire Jacques-Louis Lions, F-75005, Paris, France

mirebeau@ann.jussieu.fr 